\title{
Sign Changing Solutions for Coupled Critical Elliptic Equations
}

\author{
Xin Wang ${ }^{1}$ and Xiaorui Yue $\mathbb{D}^{2}$ \\ ${ }^{1}$ School of Science, Hainan University, Haikou 570228, China \\ ${ }^{2}$ School of Mathematics, China University of Mining and Technology, Xuzhou 221116, China \\ Correspondence should be addressed to Xiaorui Yue; yxr@hainu.edu.cn
}

Received 1 December 2019; Accepted 3 January 2020; Published 11 February 2020

Academic Editor: Guozhen Lu

Copyright ( 92020 Xin Wang and Xiaorui Yue. This is an open access article distributed under the Creative Commons Attribution License, which permits unrestricted use, distribution, and reproduction in any medium, provided the original work is properly cited.

In this paper, we consider the coupled elliptic system with a Sobolev critical exponent. We show the existence of a sign changing solution for problem $(\mathscr{P})$ for the coupling parameter $-\sqrt{\mu_{1} \mu_{2}}<\beta<0$. We also construct multiple sign changing solutions for the symmetric case.

\section{Introduction}

In this paper, we consider the following coupled elliptic system with a Sobolev critical exponent:

$$
(\mathscr{P})\left\{\begin{array}{l}
-\Delta u_{1}+\lambda_{1} u_{1}=v_{1}\left|u_{1}\right|^{p_{1}-2} u_{1}+\mu_{1}\left|u_{1}\right|^{2^{*}-2} u_{1} \\
+\beta\left|u_{1}\right|^{\left(2^{*} / 2\right)-2} u_{1}\left|u_{2}\right|^{2^{*} / 2}, \quad \text { in } \Omega, \\
-\Delta u_{2}+\lambda_{2} u_{2}=v_{2}\left|u_{2}\right|^{p_{2}-2} u_{2}+\mu_{2}\left|u_{2}\right|^{2^{*}-2} u_{2} \\
+\beta\left|u_{1}\right|^{2^{*} / 2}\left|u_{2}\right|^{\left(2^{*} / 2\right)-2} u_{2}, \quad \text { in } \Omega, \\
u_{1}=u_{2}=0 \text { on } \partial \Omega,
\end{array}\right.
$$

where $\Omega \subset \mathbb{R}^{N}$ is a bounded smooth domain, $N \geq 6$, $2<p_{j}<2^{*}, \quad \lambda_{j} \in\left(-\lambda_{1}(\Omega), 0\right), \nu_{j}, \mu_{j}>0$ for $j=1,2$, and $\lambda_{1}(\Omega)$ is the first eigenvalue of $-\Delta$ with the Dirichlet boundary condition.

In recent years, the following coupled elliptic system has attracted much interest:

$$
\begin{cases}-\Delta u_{1}+\lambda_{1} u_{1}=\mu_{1} u_{1}^{3}+\beta u_{2}^{2} u_{1}, & x \in \Omega, \\ -\Delta u_{2}+\lambda_{2} u_{2}=\mu_{2} u_{2}^{3}+\beta u_{1}^{2} u_{2}, & x \in \Omega, \\ u_{1}=u_{2}=0, & x \in \partial \Omega .\end{cases}
$$

$\Omega \subset \mathbb{R}^{N}$ is a bounded smooth domain or $\Omega=\mathbb{R}^{N}, N \geq 3$. System (2) arises when we consider the standing wave solutions of the two coupled Gross-Pitaevskii equations:

$$
\begin{cases}-i \frac{\partial}{\partial t} \Phi_{1}=\Delta \Phi_{1}+\mu_{1}\left|\Phi_{1}\right|^{2} \Phi_{1}+\beta\left|\Phi_{2}\right|^{2} \Phi_{1}, & x \in \Omega, t>0, \\ -i \frac{\partial}{\partial t} \Phi_{2}=\Delta \Phi_{2}+\mu_{2}\left|\Phi_{2}\right|^{2} \Phi_{2}+\beta\left|\Phi_{1}\right|^{2} \Phi_{2}, & x \in \Omega, t>0, \\ \Phi_{j}=\Phi_{j}(x, t) \in \mathbb{C}, & j=1,2, \\ \Phi_{j}(x, t)=0, & x \in \partial \Omega, t>0, \\ & j=1,2 .\end{cases}
$$

The system above has applications in many physical problems (see $[1,2])$. It also arises in the Hartree-Fock theory for a double condensate, which is a binary mixture of Bose-Einstein condensates in two different hyperfine states $|1\rangle$ and $|2\rangle$ (see [3] and the references therein). Considering the solitary wave solutions of system (3), we set $\Phi_{j}(x, t)=e^{i \lambda_{j} t} u_{j}(x)$ for $j=1,2$. Then, it is reduced to system (2). For the subcritical case, i.e., $N=3$, the existence of least energy and other finite energy solutions as well as the existence and multiplicity of positive and sign-changing solutions are studied in [2, 4-23] and the references therein. For the critical case, i.e., $N=4\left(2^{*}:=((2 N) /(N-2))=4\right)$, the existence of a positive least energy solution is proved when $\beta$ is negative, positive small, and positive large in [24]. For the higher dimension $N \geq 5$, the authors in $[25,26]$ also consider the following critical case: 


$$
\left\{\begin{array}{l}
-\Delta u_{1}+\lambda_{1} u_{1}=\mu_{1} u_{1}^{2^{*}-1}+\beta u_{1}^{\left(2^{*} / 2\right)-1} u_{2}^{2^{*} / 2}, \quad \text { in } \Omega, \\
-\Delta u_{2}+\lambda_{2} u_{2}=\mu_{2} u_{2}^{2^{*}-1}+\beta u_{1}^{\left(2^{*} / 2\right)} u_{2}^{\left(2^{*} / 2\right)-1}, \quad \text { in } \Omega, \\
u_{1}, u_{2} \geq 0 \text { in } \Omega, u_{1}=u_{2}=0 \text { on } \partial \Omega .
\end{array}\right.
$$

Note that when $N=4$, system (4) is the same as system (2). But interestingly, the authors in [25] find different results for the higher dimension case $N \geq 5$ from that of $N=4$. The authors in [27] also consider the even case:

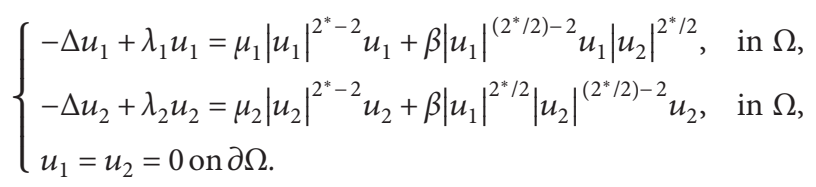

They show that when $N \geq 6$, system (5) has a sign changing solution for any $\beta<0$.

Recall that when $\beta=0$, system (4) becomes the famous Brezis-Nirenberg problem:

$$
\begin{cases}-\Delta u_{j}+\lambda_{j} u_{j}=\mu_{j} u_{j}^{2^{*}-1}, & \text { in } \Omega, \\ u_{j} \geq 0 \text { in } \Omega, u_{j}=0 \text { on } \partial \Omega, & j=1,2,\end{cases}
$$

which are two different independent scalar equations. In [28], the authors show that when $N \geq 4,-\lambda_{1}(\Omega)<\lambda_{j}<0$, and $\mu_{j}=1$, equation (6) has a ground state solution. For the even case,

$$
\begin{cases}-\Delta u+\lambda_{j} u=\mu_{j}|u|^{2^{*}-2} u, & \text { in } \Omega, \\ u=0 \text { on } \partial \Omega, & j=1,2 .\end{cases}
$$

The authors in [29] show that when $N \geq 6,-\lambda_{1}(\Omega)<\lambda_{j}<0$, and $\mu_{j}>0$, equation (7) has sign changing solutions for $j=1,2$. In [30, 31], when $N \geq 4, \lambda_{j}<0$, and $\mu_{j}>0$, the authors obtained nontrivial solutions of (7) for $j=1,2$.

In fact, in the pioneering paper [28] of Brezis and Nirenberg, they also study a more general equation including the following classical case:

$$
\begin{cases}-\Delta u+\lambda_{j} u=v_{j} u^{p_{j}-1}+\mu_{j} u^{2^{*}-1}, & \text { in } \Omega, \\ u \geq 0 \text { in } \Omega, u=0 \text { on } \partial \Omega, & j=1,2,\end{cases}
$$

where $2<p_{j}<2^{*}$ and $v_{j}>0$. From [28], we know that when $N \geq 4, v_{j}, \mu_{j}>0$, and $\lambda_{j} \in\left(-\lambda_{1}(\Omega), 0\right)$, then problem (8) has positive ground state solutions $v_{1}, v_{2} \in C^{2}(\Omega) \cap C(\bar{\Omega})$ with the energy:

$$
\begin{aligned}
0<B_{j} & :=\frac{1}{2} \int_{\Omega}\left(\left|\nabla v_{j}\right|^{2}+\lambda_{j} v_{j}^{2}\right)-\int_{\Omega} \frac{v_{j}}{p_{j}} v_{j}^{p}-\frac{1}{2^{*}} \int_{\Omega} \mu_{j} v_{j}^{2^{*}} \\
& <\frac{1}{N} \mu_{j}^{-((N-2) / 2)} S^{N / 2}, \quad j=1,2,
\end{aligned}
$$

where $S$ is the sharp imbedding constant from $D^{1,2}\left(\mathbb{R}^{N}\right)$ into $L^{2^{*}}\left(\mathbb{R}^{N}\right)$.

Based on these, firstly, we try to show similar results in [29-31] for the even case of the general equation (8), i.e.,

$$
\begin{cases}-\Delta u+\lambda_{j} u=v_{j}|u|^{p_{j}-2} u+\mu_{j}|u|^{2^{*}-2} u, & \text { in } \Omega, \\ u=0 \text { on } \partial \Omega, & j=1,2 .\end{cases}
$$

Then, naturally and interestingly, we guess that the more general system corresponding to system (5) also has similar result of the existence of sign changing solutions. That is, we consider the more general critical elliptic system as follows:

$$
\left\{\begin{array}{l}
-\Delta u_{1}+\lambda_{1} u_{1}=v_{1}\left|u_{1}\right|^{p_{1}-2} u_{1}+\mu_{1}\left|u_{1}\right|^{2^{*}-2} u_{1} \\
\quad+\beta\left|u_{1}\right|^{\left(2^{*} / 2\right)-2} u_{1}\left|u_{2}\right|^{2^{*} / 2}, \quad \text { in } \Omega, \\
-\Delta u_{2}+\lambda_{2} u_{2}=v_{2}\left|u_{2}\right|^{p_{2}-2} u_{2}+\mu_{2}\left|u_{2}\right|^{2^{*}-2} u_{2} \\
+\beta\left|u_{1}\right|^{2^{*} / 2}\left|u_{2}\right|^{\left(2^{*} / 2\right)-2} u_{2}, \quad \text { in } \Omega, \\
u_{1}=u_{2}=0 \text { on } \partial \Omega .
\end{array}\right.
$$

Precisely, we get the following results.

Theorem 1. Assume that $N \geq 6,2<p_{j}<2^{*}, \lambda_{j} \in$ $\left(-\lambda_{1}(\Omega), 0\right)$, and $\nu_{j}, \mu_{j}>0$, for $j=1,2$; then, (10) has sign changing solutions for $j=1,2$ and the energy is the least one among all sign changing solutions.

Then, we can consider the case $\lambda_{1}=\lambda_{2}=\lambda$ and $p_{1}=$ $p_{2}=p$. The following equation is one case of (10):

$$
\left\{\begin{array}{l}
-\Delta u+\lambda u=v|u|^{p-2} u+|u|^{2^{*}-2} u, \text { in } \Omega, \\
u=0 \text { on } \partial \Omega,
\end{array}\right.
$$

where $v>0$. Let $v$ and $w$ be the sign changing solutions of (12) when $N \geq 6, \lambda \in\left(-\lambda_{1}(\Omega), 0\right)$, and $N \geq 4, \lambda \leq-\lambda_{1}(\Omega)$, respectively (the latter case really exists and we shall show it later). By Lemma 1 in [25], the system below has a positive solution:

$$
\left\{\begin{array}{l}
\mu_{1} a_{1}^{2^{*}-2}+\beta a_{1}^{\left(2^{*} / 2\right)-2} a_{2}^{2^{*} / 2}=1, \\
\mu_{2} a_{2}^{2^{*}-2}+\beta a_{1}^{2^{*} / 2} a_{2}^{\left(2^{*} / 2\right)-2}=1 .
\end{array}\right.
$$

Then, we can construct solutions of (11) by using $v$ and $w$ as in $[2,24,25]$.

Theorem 2. Let $\left(a_{1}, a_{2}\right)$ be a solution of (13). Assume that $\nu_{1}=v / a_{1}^{p-2}$ and $\nu_{2}=v / a_{2}^{p-2}, \mu_{1}, \mu_{2}, \beta>0$ and one of the following happens:

(1) $N \geq 6,-\lambda_{1}(\Omega)<\lambda_{1}=\lambda_{2}<0$,

(2) $N \geq 4, \lambda_{1}=\lambda_{2} \leq-\lambda_{1}(\Omega)$,

Then $\left(a_{1} v, a_{2} v\right)$ and $\left(a_{1} w, a_{2} w\right)$ are sign changing solutions of system (11).

We also get a least energy semi-sign changing solution for system (11).

Theorem 3. Assume that $N \geq 6,2<p_{j}<2^{*},-\lambda_{1}(\Omega)<\lambda_{j}<0$, $v_{j}, \mu_{j}>0$, and $-\sqrt{\mu_{1} \mu_{2}}<\beta<0$. Then, system (11) has a semisign changing solution with one component changing sign and the other one positive, and the energy is the least one among all these kinds of solutions. 
Next, we give the proof of Theorem 1 in Section 2. And we shall show Theorems 2 and 3 in Sections 3 and 4, respectively.

\section{Proof of Theorem 1}

In this section, we consider scalar case (10). Fixing $j=1$ or 2 , we show the existence result. The proof is similar to that in [29]. Considering we need this result in the following two Theorems 2 and 3, we verify it also for the completeness of the current paper. The working space and some notations shall be given firstly. We assume that all the integrations below are taken over $\Omega$ if without special specification. Since $\lambda_{j}>-\lambda_{1}(\Omega)$, we can define the equivalent inner product in $H_{0}^{1}(\Omega)$ by

$$
(u, v)_{j}=\int\langle\nabla u, \nabla v\rangle+\lambda_{j} u v
$$

which gives rise to a norm denoted by $\|\cdot\|_{j}$. We also use $|u|_{q}=\left(\int_{\Omega}|u|^{q} \mathrm{~d} x\right)^{1 / q}(1 \leq q<\infty)$ and $\|u\|_{0}=\left(\int_{\Omega}|\nabla u|^{2} \mathrm{~d} x\right)^{1 / 2}$ for convenience. Then, the energy functional of equation (10) is

$$
I_{j}(u)=\frac{1}{2}\|u\|_{j}^{2}-\frac{1}{p_{j}} \int v_{j}\left|u_{j}\right|^{p_{j}}-\frac{1}{2^{*}} \int \mu_{j}\left|u_{j}\right|^{2^{*}} .
$$

Recall $v_{j}$ is a ground state solution of (8), that is,

$$
B_{j}:=I_{j}\left(v_{j}\right)=\inf \left\{I_{j}(u): u \in H_{0}^{1}(\Omega) \backslash\{0\}, I_{j}^{\prime}(u) u=0\right\} .
$$

For the sign changing case, we define the manifold as follows:

$$
\mathcal{S}_{j}:=\left\{u \in H_{0}^{1}(\Omega): u^{ \pm} \equiv 0, I_{j}^{\prime}\left(u^{ \pm}\right) u^{ \pm}=0\right\} .
$$

where $u^{ \pm}:= \pm \max \{u, 0\}$ and the condition in the definition of (17) is that

$$
\left\|u^{ \pm}\right\|_{j}^{2}=v_{j} \int\left|u^{ \pm}\right|^{p_{j}}+\mu_{j} \int\left|u^{ \pm}\right|^{2^{*}}
$$

It is easy to check that $\mathcal{S}_{j} \neq \varnothing$. Then, we define

$$
\mathscr{B}_{j}:=\inf _{u \in \mathcal{S}_{j}} I_{j}(u) \text {. }
$$

We need a conclusion in [32].

Lemma 1 (see [32]). Consider a rectangle $\mathbf{R}=\Pi_{1}^{s}\left[a_{i}, b_{i}\right] \subset \mathbb{R}^{s}$ and a continuous function $\boldsymbol{\Phi}=\left(\boldsymbol{\Phi}_{1}, \ldots, \boldsymbol{\Phi}_{s}\right): \mathbf{R} \longrightarrow \mathbb{R}^{s}$. If $\left.\Phi_{i}\right|_{x_{i}=a_{i}}>0>\left.\Phi_{i}\right|_{x_{i}=b_{i}}$ hold for all $i=1, \ldots, s$, then $\Phi$ has a zero inside $\mathbf{R}$.

Similarly as in [29], we set

$$
E_{j}(u):= \begin{cases}0, & u=0, \\ \frac{v_{j} \int|u|^{p_{j}}+\mu_{j} \int|u|^{2^{*}}}{\|u\|_{j}^{2}}, & u \neq 0 .\end{cases}
$$

Then, (18) is equivalent to $E_{j}\left(u^{+}\right)=E_{j}\left(u^{-}\right)=1$. Define

$$
\begin{aligned}
\mathscr{H}_{j}:= & \left\{h \in C\left([0,1] \times[0,1], H_{0}^{1}(\Omega)\right): h(t, 0)=0,\right. \\
& h(0, s) \geq 0, h(1, s) \leq 0, I_{j}(h(t, 1)) \leq 0, \\
& \left.E_{j}(h(s, 1)) \geq 2, \quad \forall t, s \in[0,1]\right\} .
\end{aligned}
$$

Since $\mu_{j}>0$, it is easy to see that $\mathscr{H}_{j} \neq \varnothing$. Then, we have the following lemma.

Lemma 2. $\mathscr{B}_{j}=\inf _{h \in \mathscr{H}_{j}} \sup _{u \in h([0,1] \times[0,1])} I_{j}(u)$.

Proof. For any $u \in \mathcal{S}_{j}, t, s \geq 0$, we have

$$
\begin{aligned}
I_{j}\left(t u^{+}-s u^{-}\right)= & {\left[\frac{1}{2} t^{2}\left\|u^{+}\right\|_{j}^{2}-\frac{v_{j}}{p_{j}} t^{p_{j}} \int\left|u^{+}\right|^{p_{j}}-\frac{\mu_{j}}{2^{*}} t^{2^{*}} \int\left|u^{+}\right|^{2^{*}}\right] } \\
& +\left[\frac{1}{2} s^{2}\left\|u^{-}\right\|_{j}^{2}-\frac{v_{j}}{p_{j}} s^{p_{j}} \int\left|u^{-}\right|^{p_{j}}-\frac{\mu_{j}}{2^{*}} s^{2^{*}} \int\left|u^{-}\right|^{2^{*}}\right] \\
:= & f_{+}(t)+f_{-}(s) .
\end{aligned}
$$

Then,

$$
\sup _{t, s \geq 0} I_{j}\left(t u^{+}-s u^{-}\right)=f_{+}(1)+f_{-}(1)=I_{j}(u) .
$$

Denote the map $h \in \mathscr{H}_{j}$ satisfying

$$
h([0,1] \times[0,1]) \subset\left\{t u^{+}-s u^{-}: t, s \geq 0\right\} .
$$

By (23), we have

$$
\mathscr{B}_{j} \geq \inf _{h \in \mathscr{H}_{j}} \sup _{u \in h([0,1] \times[0,1])} I_{j}(u) .
$$

On the other hand, for any $h \in \mathscr{H}_{j}$, we have

$$
\begin{aligned}
E_{j}\left(h(\mathbf{t})^{+}\right)-E_{j}\left(h(\mathbf{t})^{-}\right) \begin{cases}\geq 0, & \mathbf{t} \in\{(0, s): s \in[0,1]\}, \\
\leq 0, & \mathbf{t} \in\{(1, s): s \in[0,1]\},\end{cases} \\
E_{j}\left(h(\mathbf{t})^{+}\right)+E_{j}\left(h(\mathbf{t})^{-}\right)-2 \begin{cases}<0, & \mathbf{t} \in\{(t, 0): t \in[0,1]\}, \\
\geq 0, & \mathbf{t} \in\{(t, 1): t \in[0,1]\} .\end{cases}
\end{aligned}
$$

Then, by Lemma 1 , we have that there exists $t_{0} \in[0,1] \times$ $[0,1]$ such that $E_{j}\left(h\left(\mathbf{t}_{0}\right)^{+}\right)=E_{j}\left(h\left(\mathbf{t}_{0}\right)^{-}\right)=1$, i.e., $u_{0}:=$ $h\left(\mathbf{t}_{0}\right) \in \mathcal{S}_{j}$. Consequently,

$$
\mathscr{B}_{j} \leq \inf _{h \in \mathscr{H}_{j}} \sup _{u \in h([0,1] \times[0,1])} I_{j}(u) .
$$

This completes the proof.

Now, we have an upper estimate for $\mathscr{B}_{j}$.

Lemma 3. $\mathscr{B}_{j}<c_{j}^{*}:=B_{j}+(1 / N) \mu_{j}^{-((N-2) / 2)} S^{N / 2}$.

Proof. Since $v_{j} \in C(\bar{\Omega})$ and $v_{j}=0$ on $\partial \Omega$, there exists $B\left(x_{0}, 2 r_{0}\right):=\left\{x:\left|x-x_{0}\right| \leq 2 r\right\} \subset \Omega$ for some $x_{0}$ near $\partial \Omega$ and $r>0$ small to be decided later. Take a cutoff function $\phi \in C_{0}^{1}\left(B\left(x_{0}, 2 r\right)\right)$ with $0 \leq \phi \leq 1$ and $\phi(x) \equiv 1$ for $\left|x-x_{0}\right| \leq r$. Let 


$$
U_{\varepsilon, x_{0}}(x):=\frac{\left[N(N-2) \varepsilon^{2}\right]^{(N-2) / 4}}{\left[\mathcal{E}^{2}+\left|x-x_{0}\right|^{2}\right]^{(N-2) / 2}}, \quad x \in \mathbb{R}^{N} .
$$

Then (see $[33,34]), U_{\varepsilon, x_{0}}$ is the solution of the following equation:

$$
-\Delta u=|u|^{2^{*}-2} u, \quad u \in D^{1,2}\left(\mathbb{R}^{N}\right) .
$$

Set $u_{\varepsilon}:=\phi U_{\varepsilon, x_{0}}$; then (see [35]),

$$
\begin{gathered}
\int\left|\nabla u_{\varepsilon}\right|^{2} \mid=S^{N / 2}+O\left(\varepsilon^{N-2}\right), \\
\int\left|u_{\varepsilon}\right|^{2^{*}}=S^{N / 2}+O\left(\varepsilon^{N}\right), \\
\int\left|u_{\varepsilon}\right|^{2} \geq C \varepsilon^{2}+O\left(\varepsilon^{N-2}\right), \\
\int\left|u_{\varepsilon}\right| \leq C \varepsilon^{(N-2 / 2)}, \\
\int\left|u_{\varepsilon}\right|^{p_{j}-1} \leq C \varepsilon^{(N-2)\left(3-p_{j}\right)+4 / 2}, \\
\int\left|u_{\varepsilon}\right|^{2^{*}-1} \leq C \varepsilon^{(N-2) / 2} .
\end{gathered}
$$

Now, we show that

$$
\sup _{t, s \in \mathbb{R}} I_{j}\left(t v_{j}+s u_{\varepsilon}\right)<c_{j}^{*} .
$$

In fact, by $(30)$ and $\left((N-2)\left(3-p_{j}\right)+4\right) /(2)>(N-2)$ /(2) since $p_{j}<2^{*}$, we have

$$
\begin{aligned}
I_{j}\left(t v_{j}+s u_{\varepsilon}\right) \leq & {\left[\frac{1}{2} t^{2}\left\|v_{j}\right\|_{j}^{2}-\frac{v_{j}}{p_{j}} t_{j} \int\left|v_{j}\right|^{p_{j}}-\frac{\mu_{j}}{2^{*}} t^{2^{*}} \int\left|v_{j}\right|^{2^{*}}\right] } \\
& +\left[\frac{1}{2} s^{2}\left\|u_{\varepsilon}\right\|_{j}^{2}-\frac{\mu_{j}}{2^{*}} s^{2^{*}} \int\left|u_{\varepsilon}\right|^{2^{*}}\right] \\
& +C_{1}\left[|t s|\left|\Delta v_{j}\right|_{L^{\infty}\left(B\left(x_{0}, 2 r\right)\right)}\left|u_{\varepsilon}\right|_{1}\right. \\
& \left.+|t s|\left|v_{j}\right|_{L^{\infty}\left(B\left(x_{0}, 2 r\right)\right)}\left|u_{\varepsilon}\right|_{1}\right] \\
& +C_{2}\left[\left|v_{j}\right|_{L^{\infty}\left(B\left(x_{0}, 2 r\right)\right)}\left(\int\left|s u_{\varepsilon}\right|^{p_{j}-1}+\int\left|s u_{\varepsilon}\right|^{2^{*}-1}\right)\right] \\
\leq & B_{j}+\frac{1}{N} \mu_{j}^{-((N-2)) / 2} S^{N / 2} \\
& +C_{3}\left|v_{j}\right|_{L^{\infty}\left(B\left(x_{0}, 2 r\right)\right)} \varepsilon^{(N-2) / 2}-C_{4} \varepsilon^{2} \\
< & B_{j}+\frac{1}{N} \mu^{-((N-2) / 2)} S^{N / 2},
\end{aligned}
$$

for $r>0$ small enough.

Then by Lemma 2, the conclusion follows.

Set

$$
\mathscr{G}:=\left\{u \in H_{0}^{1}(\Omega):\left|E_{j}\left(u^{ \pm}\right)-1\right|<\frac{1}{2}\right\} .
$$

Then, we show that $I_{j}$ satisfies a local (PS) condition in the following sense.
Lemma 4. Under the assumptions in Theorem 1, for any $c<c^{*}$, if $\left\{u_{n}\right\} \subset \mathscr{G}$ satisfies that

$$
\begin{aligned}
I_{j}\left(u_{n}\right) & \longrightarrow c, \\
I^{\prime}\left(u_{n}\right) & \longrightarrow 0, \\
\quad \text { as } n & \longrightarrow \infty,
\end{aligned}
$$

then we have that $\left\{u_{n}\right\}$ has a convergent subsequence in $H_{0}^{1}(\Omega)$.

Proof. It is easy to check that $\left\{u_{n}\right\}$ is bounded in $H_{0}^{1}(\Omega)$, so there exists $u \in H_{0}^{1}(\Omega)$ such that $u_{n} \rightarrow \mathcal{u}$ weakly in $H_{0}^{1}(\Omega)$ up to a subsequence and $u_{n} \longrightarrow u$ strongly in $L^{q}(\Omega)$ for $1 \leq q<2^{*}$. Then, $I_{j}^{\prime}(u)=0$ and $u_{n}^{ \pm} \rightarrow \mathcal{u}^{ \pm}$weakly in $H_{0}^{1}(\Omega)$. By the Sobolev inequality, we have that there exist positive constants $C_{0}$ and $C_{1}$ independent of $n$ such that $\left\|u_{n}^{ \pm}\right\|_{j} \geq C_{0}>0$ and then

$$
\begin{aligned}
I_{j}\left(u_{n}\right) & =I_{j}\left(u_{n}\right)-\frac{1}{p_{j}} I_{j}^{\prime}\left(u_{n}\right) u_{n}+o(1) \\
& =\left(\frac{1}{2}-\frac{1}{p_{j}}\right)\left\|u_{n}\right\|_{j}^{2}+\left(\frac{1}{p_{j}}-\frac{1}{2^{*}}\right) \mu_{j} \int\left|u_{n}\right|^{2^{*}} \geq C_{1}>0 .
\end{aligned}
$$

Since

$$
\begin{aligned}
o(1) & =\left(I_{j}^{\prime}\left(u_{n}\right)-I_{j}^{\prime}(u)\right)\left(u_{n}^{ \pm}-u^{ \pm}\right) \\
& =\left\|u_{n}^{ \pm}-u^{ \pm}\right\|_{0}^{2}-\mu_{j} \int\left|u_{n}^{ \pm}-u^{ \pm}\right|^{2^{*}}+o(1) \\
& \geq\left\|u_{n}^{ \pm}-u^{ \pm}\right\|_{0}^{2}\left(1-S^{(N /(N-2))} \mu_{j}\left\|u_{n}^{ \pm}-u^{ \pm}\right\|_{j}^{2^{*}-2}\right)+o(1),
\end{aligned}
$$

we have either $u_{n}^{ \pm} \longrightarrow u^{ \pm}$strongly in $H_{0}^{1}(\Omega)$ or $\left\|u_{n}^{ \pm}-u^{ \pm}\right\|_{0}^{2} \geq$ $\mu_{j}^{(N-2) / 2} S^{N / 2}$. Considering the latter case, we have

$$
\begin{aligned}
I_{j}\left(u_{n}^{ \pm}\right) & =I_{j}\left(u_{n}^{ \pm}-u^{ \pm}\right)+I_{j}\left(u^{ \pm}\right)+o(1) \\
& =\frac{1}{N}\left\|u_{n}^{ \pm}-u^{ \pm}\right\|_{0}^{2}+I_{j}\left(u^{ \pm}\right)+o(1) \\
& \geq \frac{1}{N} \mu_{j}^{-((N-2) / 2)} S^{N / 2}+o(1) .
\end{aligned}
$$

Therefore,

$$
\begin{aligned}
& c_{j}^{*}>\lim _{n \longrightarrow \infty} I_{j}\left(u_{n}\right)=\lim _{n \longrightarrow \infty}\left[I_{j}\left(u_{n}^{+}\right)+I_{j}\left(u_{n}^{-}\right)\right] \\
& \geq B_{j}+\frac{1}{N} \mu_{J}^{-((N-2) / 2)} S^{N / 2}=c_{j}^{*},
\end{aligned}
$$

is a contradiction. That is, we must have $u_{n} \longrightarrow u$ strongly in $H_{0}^{1}(\Omega)$ and $u^{ \pm} \neq 0$.

Now we are ready to show Theorem 1 based on a deformation lemma (see [36-38] for instance).

Proof of Theorem 1. Obviously, $I_{j}$ is coercive on $\delta_{j}$ and then there exists a minimizing sequence $\left\{u_{n}\right\} \subset \mathcal{S}_{j}$. Let $h_{n} \in \mathscr{H}_{j}$ such that 


$$
h_{n}([0,1] \times[0,1]) \subset\left\{t u_{n}^{+}-s u_{n}^{-}: t, s \geq 0\right\} .
$$

Then, by Lemma 2, we have

$$
\lim _{n \longrightarrow \infty} \sup _{u \in h_{n}([0,1] \times[0,1])} I_{j}(u)=\lim _{n \longrightarrow \infty} I_{j}\left(u_{n}\right)=\mathscr{B}_{j} .
$$

By the well-known deformation lemma, we have that there exists a sequence $\left\{\hat{u}_{n}\right\} \subset H_{0}^{1}(\Omega)$ such that

$$
\begin{array}{r}
\operatorname{dist}\left(\hat{u}_{n}, h_{n}([0,1] \times[0,1])\right) \longrightarrow 0, \\
I_{j}\left(\hat{u}_{n}\right) \longrightarrow \mathscr{B}_{j}, I_{j}^{\prime}\left(\hat{u}_{n}\right) \longrightarrow 0, \text { as } n \longrightarrow \infty .
\end{array}
$$

Obviously, $\left\{\hat{u}_{n}\right\}$ is bounded and there exists $\hat{u} \in H_{0}^{1}(\Omega)$ such that $\hat{u}_{n} \rightarrow \hat{u}$ weakly in $H_{0}^{1}(\Omega)$; then, $I_{j}^{\prime}(\hat{u})=0$ by the definition of weak convergence.

Now, we claim that $\left\{\hat{u}_{n}\right\} \subset \mathscr{G}$. In fact, by (41), there exists a sequence $\left\{\tilde{u}_{n}\right\}$ with

$$
\begin{array}{r}
\widetilde{u}_{n}=t_{n} u_{n}^{+}-s_{n} u_{n}^{-} \in h_{n}([0,1] \times[0,1]), \\
\operatorname{dist}\left(\hat{u}_{n}, \widetilde{u}_{n}\right) \longrightarrow 0 .
\end{array}
$$

Then, for $n$ large enough depending on $\xi>0$,

$$
\begin{aligned}
& I_{j}\left(\tilde{u}_{n}^{+}\right)=I_{j}\left(t_{n} u_{n}^{+}\right)>I_{j}\left(u_{n}^{+}\right)-\xi \geq B_{j}-\xi, \\
& I_{j}\left(\tilde{u}_{n}^{-}\right)=I_{j}\left(s_{n} u_{n}^{-}\right)>I_{j}\left(u_{n}^{-}\right)-\xi \geq B_{j}-\xi .
\end{aligned}
$$

(44), (45), and (43) implies that $\hat{u}_{n}^{ \pm} \neq 0$. Since $I_{j}^{\prime}\left(\hat{u}_{n}\right) \hat{u}_{n}^{ \pm}=$ $o(1)$, the claim comes true.

Therefore, by Lemmas 3 and 4 , we have that $\hat{u}_{n} \longrightarrow \hat{u}$ strongly in $H_{0}^{1}(\Omega)$ with $\hat{u}^{ \pm} \neq 0$, i.e., $\hat{u}$ is a sign changing solution of equation (10) and the energy $I_{j}(\hat{u})$ is the least one among all sign changing solutions of (10).

\section{Proof of Theorem 2}

Firstly, inspired by $[30,31]$, we show the following theorem about the scalar equation by the minimax method (see [39]), and we would like to just give the sketch for the completeness of the current paper. We also use the same notations as in Section 2 whenever no confusion arises.

Denote $\varphi_{1}, \varphi_{2}, \varphi_{3}, \ldots$ as the $L^{2}$ normalized eigenfunctions of $-\Delta$, corresponding to positive eigenvalues $0<\lambda_{1}(\Omega)<\lambda_{2}(\Omega) \leq \lambda_{3}(\Omega) \leq \ldots \uparrow+\infty$, counted with their multiplicity. We set $H_{k}:=\left\langle\varphi_{1}, \varphi_{2}, \ldots, \varphi_{k}\right\rangle$ as the $k$ dimensional space in $H_{0}^{1}(\Omega)$. For the fixed $\lambda_{j}<0$, define

$$
\lambda_{j}^{+}:=\min \left\{\lambda_{k}(\Omega):-\lambda_{j}<\lambda_{k}(\Omega)\right\} .
$$

We may suppose that $\lambda_{j}^{+}=\lambda_{k_{0}}(\Omega)$ for some $k_{0} \in \mathbb{N}$. Especially, we set $\lambda_{0}(\Omega) \equiv 0$.

Theorem 4. Assume that $N \geq 4,2<p_{j}<2^{*}, \lambda_{j}<0$, and $v_{j}$, $\mu_{j}>0$, for $j=1,2$, then equation (10) has a nontrivial solution. Moreover, when $\lambda_{j} \leq-\lambda_{1}(\Omega)$, the solution changes sign.
Proof. It is easy to check that $I_{j}$ satisfies the following geometry.

(a) For $u \in\left(H_{k_{0}-1}\right)^{\perp}$, there exist $r_{0}, \alpha_{0}>0$ such that $I_{j}(u)>\alpha_{0}$ with $\|u\|_{0}=r_{0}$ and $I_{j}(u)>0$ for $0<\|u\|_{0}<r_{0}$

(b) For any finite dimensional subspace $\widetilde{H} \subset H_{0}^{1}(\Omega)$, it holds that $\hat{I}_{j}^{0} \cap \widetilde{H}$ is bounded in $H_{0}^{1}(\Omega)$, where $\hat{I}_{j}^{0}:=\left\{u \in H_{0}^{1}(\Omega): I_{j}(u) \geq 0\right\}$

As in [36], we define the minimax value:

$$
\hat{\boldsymbol{c}}_{j}:=\sup _{h \in \Lambda} \inf _{u \in B_{1} \cap\left(H_{k_{0}-1}\right)^{\perp}} I_{j}(h(u))
$$

where

$$
\begin{aligned}
\Lambda:=\left\{h \in C\left(H_{0}^{1}(\Omega), H_{0}^{1}(\Omega)\right): h\left(\operatorname{Int} B_{1}\right) \subset \hat{I}_{j}^{0} \cup B_{r_{0}},\right. \\
\left.\quad h \text { is an odd homeomorphism of } H_{0}^{1}(\Omega) \text { onto } H_{0}^{1}(\Omega)\right\} .
\end{aligned}
$$

Obviously, $h=r_{0}$ id $\in \Lambda$. We also set another minimax value:

$$
\hat{b}_{j}:=\inf _{K \in \hat{\Lambda}} \sup _{u \in K} I_{j}(u)
$$

where

$$
\begin{gathered}
\hat{\Lambda}:=\left\{K \subset H_{0}^{1}(\Omega): K\right. \text { is compact, symmetric, and } \\
\left.\gamma\left(K \cap h\left(\partial B_{1}\right)\right) \geq k_{0}, \text { for all } h \in \Lambda\right\},
\end{gathered}
$$

and $\gamma$ is the Krasnoselskii genus. It can be verified that $\hat{\Lambda} \neq \varnothing$. By properties (a) and (b), we can show that

$$
0<\alpha_{0} \leq \hat{c}_{j} \leq \hat{b}_{j}<\hat{c}_{j}^{*}:=\frac{1}{N} \mu_{j}^{-((N-2) / 2)} S^{N / 2},
$$

and $I_{j}$ satisfies the $(\mathrm{PS})_{\hat{c}_{j}}$ condition. Also by a deformation lemma, there exists a (PS $)_{\hat{c}_{j}}$ sequence $\left\{u_{n}\right\}$ of $I_{j}$ and then $u_{n} \longrightarrow u$ in $H_{0}^{1}(\Omega)$. That is, $u$ is a nontrivial solution of equation (10).

Multiply the equations in (10) with the first eigenfunction $\varphi_{1}$ and integrate over $\Omega$; we have

$$
\left(\lambda_{1}(\Omega)+\lambda_{j}\right) \int_{\Omega} u \varphi_{1}=\int_{\Omega}\left(v_{j}|u|^{p_{j}-2} u \varphi_{1}+\mu_{j}|u|^{2^{*}-2} u_{2} \varphi_{1}\right) .
$$

Then, it is easy to see that when $\lambda_{j} \leq-\lambda_{1}(\Omega), v_{j}, \mu_{j}>0$ any solution of (10) must change sign.

Proof of Theorem 2. The proof is by direct computation. Recall that $v$ and $w$ are sign changing solutions of (12) being well defined. Since $\left(a_{1}, a_{2}\right)$ satisfy $(13)$, for any $\varphi \in C_{0}^{\infty}(\Omega)$, we have

$$
\begin{aligned}
& v_{1} \int\left|a_{1} v\right|^{p-2} a_{1} v \varphi+\mu_{1} \int\left|a_{1} v\right|^{2^{*}-2} a_{1} v \varphi \\
& \quad+\beta \int\left|a_{1} v\right|^{\left.2^{*} / 2\right)-2} a_{1} v\left|a_{2} v\right|^{2^{*} / 2} \varphi \\
& =a_{1}\left(v \int|v|^{p-2} v \phi+\int|v|^{2^{*}-2} v\right)=\left(a_{1} v, \varphi\right)_{1},
\end{aligned}
$$


That is, $\left(a_{1} v, a_{2} v\right)$ satisfy the first equation in system (11). Similarly, we can verify that Theorem 2 holds.

\section{Proof of Theorem 3}

Inspired by [27, 29], we now consider the general case of system (11), i.e., we do not assume that $\lambda_{1}=\lambda_{2}$. We always assume that $\beta<0$ and $\nu_{j}, \mu_{j}>0$ for $j=1,2$. Firstly, we introduce the product space $\mathbf{H}:=H_{0}^{1}(\Omega) \times H_{0}^{1}(\Omega)$ as the working space. Define the inner product as

$$
(\mathbf{u}, \mathbf{v})=\sum_{j=1}^{2}\left(u_{j}, v_{j}\right)_{j}, \quad \text { for } \mathbf{u}=\left(u_{1}, u_{2}\right), \mathbf{v}=\left(v_{1}, v_{2}\right) \in \mathbf{H},
$$

which gives rise to a norm on $\mathbf{H}$ denoted by $\|\cdot\|$. Recall that solutions of (11) are critical points of the following energy functional:

$$
I(\mathbf{u})=\frac{1}{2}\|\mathbf{u}\|^{2}-\int \sum_{j=1}^{2} \frac{v_{j}}{p}\left|u_{j}\right|^{p_{j}}-\frac{1}{2^{*}} \int \sum_{j=1}^{2}\left(\mu_{j}\left|u_{j}\right|^{2^{*}}+2 \beta\left|u_{1}\right|^{2^{*} / 2}\left|u_{2}\right|^{2^{*} / 2}\right) .
$$

For $a, b \in \mathbb{R}$ with $a \leq b$, we denote

$$
\begin{aligned}
I^{b} & :=\{\mathbf{u} \in \mathbf{H}: I(\mathbf{u}) \leq b\}, \\
\hat{I}^{a} & :=\{\mathbf{u} \in \mathbf{H}: I(\mathbf{u}) \geq a\}, \\
I^{-1}([a, b]) & :=I^{b} \cap \hat{I}^{a} .
\end{aligned}
$$

Similar to $\delta_{j}$ related to the scalar equation, we define

$$
\begin{aligned}
\mathcal{S} & :=\left\{\left(u_{1}, u_{2}\right) \in \mathbf{H}: u_{1}^{ \pm}, u_{2} \equiv 0, I^{\prime}\left(u_{1}^{ \pm}, u_{2}\right)\left(u_{1}^{ \pm}, 0\right)\right. \\
& \left.=I^{\prime}\left(u_{1}, u_{2}\right)\left(0, u_{2}\right)=0\right\} .
\end{aligned}
$$

That is,

$$
\begin{aligned}
\left\|u_{1}^{ \pm}\right\|_{1}^{2} & =\int\left(v_{1}\left|u_{1}^{ \pm}\right|^{p_{1}}+\mu_{1}\left|u_{1}^{ \pm}\right|^{2^{*}}+\beta\left|u_{1}^{ \pm}\right|^{2^{*} / 2}\left|u_{2}\right|^{2^{*} / 2}\right), \\
\left\|u_{2}\right\|_{2}^{2} & =\int\left(v_{2}\left|u_{2}\right|^{p_{2}}+\mu_{2}\left|u_{1}\right|^{2^{*}}+\beta\left|u_{1}\right|^{2^{*} / 2}\left|u_{2}\right|^{2^{*} / 2}\right) .
\end{aligned}
$$

Let $U_{1}, U_{2} \in C_{0}^{\infty}(\Omega) \quad$ with $\quad U_{1}^{ \pm}, U_{2} \equiv 0 \quad$ and $\operatorname{supp} U_{1} \cap \operatorname{supp} U_{2}=\varnothing$, it is easy to see that there exist $t_{1}, t_{2}, s>0$ such that $\left(t_{1} U_{1}^{+}-t_{2} U_{2}^{-}, s U_{2}\right) \in \mathcal{S}$. Thus, $\mathcal{S} \neq \varnothing$. By Sobolev inequality, it is easy to see that for any $\mathbf{u} \in \mathcal{S}$, there exists a constant $C_{0}>0$ such that

$$
\left\|u_{1}^{ \pm}\right\|_{1},\left\|u_{2}\right\|_{2^{\prime}},\left|u_{1}^{ \pm}\right|_{2^{*}},\left|u_{2}\right|_{2^{*}} \geq C_{0}>0 .
$$

Set

$$
3 \varepsilon_{0}:=\min \left\{\left(\frac{1}{2}-\frac{1}{p_{1}}\right) C_{0}^{2},\left(\frac{1}{2}-\frac{1}{p_{1}}\right) C_{0}^{2}\right\} .
$$

Then, we define

$$
\mathscr{B}:=\inf _{u \in \mathcal{S}} I(\mathbf{u}) .
$$

We firstly have a lower bound for $\mathscr{B}$.

Lemma 5. Assume that $-\sqrt{\mu_{1} \mu_{2}} \leq \beta<0$; then $I$ is coercive on $\mathcal{S}$ and $\mathscr{B}>0$.
Proof. We may assume that $p_{1} \geq p_{2}$. For $\mathbf{u} \in \mathcal{S}$, note that for $\beta \geq-\sqrt{\mu_{1} \mu_{2}}$, it holds that

$$
\int \sum_{j} \mu_{j}|u|_{j}^{2^{*}}+2 \beta\left|u_{1}\right|^{2^{*} / 2}\left|u_{2}\right|^{2^{*} / 2} \geq 0
$$

Then, we have

$$
\begin{aligned}
I(\mathbf{u}) & =I(\mathbf{u})-\frac{1}{p_{2}} I^{\prime}(\mathbf{u})\left(u_{1}, u_{2}\right) \\
& =\left(\frac{1}{2}-\frac{1}{p_{2}}\right)\left(\left\|\mathbf{u}^{2}\right\|\right)+\left(\frac{1}{p_{2}}-\frac{1}{p_{1}}\right) \int v_{1}\left|u_{1}\right|^{p_{1}} \\
& +\left(\frac{1}{p_{2}}-\frac{1}{2^{*}}\right) \int\left(\sum_{j} \mu_{j}\left|u_{j}\right|_{j}^{2^{*}}+2 \beta\left|u_{1}\right|^{2^{*} / 2}\left\|u_{2}\right\|^{2^{*} / 2}\right) \\
& \geq\left(\frac{1}{2}-\frac{1}{p_{2}}\right)\|\mathbf{u}\|^{2} .
\end{aligned}
$$

(63) implies that $I$ is coercive on $\mathcal{S}$. Then, by (59), we have $\mathscr{B}>0$.

We shall give an upper bound for $\mathscr{B}$ later by defining another manifold and the infimum on it. The idea is similar to that in [27], but since the corresponding equation (10) is different and more complex as well as system (11), some new tricks should be used in the current paper, so we shall give the details of the proofs. Let

$$
\begin{aligned}
\mathscr{M} & :=\left\{\left(u_{1}, u_{2}\right) \in \mathbf{H}: u_{1}, u_{2} \equiv 0, I^{\prime}\left(u_{1}, u_{2}\right)\left(u_{1}, 0\right)\right. \\
& \left.=I^{\prime}\left(u_{1}, u_{2}\right)\left(0, u_{2}\right)=0\right\} .
\end{aligned}
$$

Obviously, $\mathcal{S} \subset \mathscr{M}$. Then, we define

$$
B:=\inf _{u \in \mathscr{M}} I(\mathbf{u}) \text {. }
$$

Before the estimate for the bound of $\mathscr{B}$, we need some preliminaries.

Lemma 6. Assume that $-\sqrt{\mu_{1} \mu_{2}} \leq \beta<0$; then, for any $\mathbf{u} \in \mathcal{S}$, $\sup _{t_{1}, t_{2}, s \geq 0} I\left(t_{1} u_{1}^{+}-t_{2} u_{1}^{-}, s u_{2}\right)$ is attained only by $t_{1}=t_{2}=s=1$. Similarly, for any $\mathbf{u} \in \mathscr{M}$, $\sup _{t, s>0} I\left(t u_{1}, s u_{2}\right)$ is attained only by $t=s=1$.

Proof. For $\mathbf{u} \in \mathcal{S}, t_{1}, t_{2}, s \geq 0$; set

$$
F\left(t_{1}, t_{2}, s\right):=I\left(t_{1} u_{1}^{+}-t_{2} u_{1}^{-}, s u_{2}\right)
$$

$$
\begin{aligned}
= & \frac{1}{2}\left(t_{1}^{2}\left\|u^{+}\right\|_{1}^{2}+t_{2}^{2}\left\|u^{-}\right\|_{1}^{2}+s^{2}\left\|u_{2}\right\|_{2}^{2}\right) \\
& -\left(\int \frac{\nu_{1}}{p_{1}} t_{1}^{p_{1}}\left|u_{1}^{+}\right|^{p}+\frac{\nu_{1}}{p_{1}} t_{2}^{p_{1}}\left|u_{1}^{-}\right|^{p_{1}}+\frac{\nu_{1}}{p_{2}} s^{p_{2}}\left|u_{2}\right|^{p_{2}}\right) \\
& -\frac{1}{2^{*}} \int\left(\mu_{1} t_{1}^{2^{*}}\left\|u_{1}^{+}\right\|^{2^{*}}+\mu_{1} t_{2}^{2^{*}}\left|u_{1}^{-}\right|^{2^{*}}+\mu_{2} s^{2^{*}}\left|u_{2}\right|^{2^{*}}\right) \\
& -\frac{2 \beta}{2^{*}} s^{2^{*} / 2} \int\left(t_{1}^{2^{*} / 2}\left|u_{1}^{+}\right|^{2^{*} / 2}+t_{2}^{2^{*} / 2}\left|u_{1}^{-}\right|^{2^{*} / 2}\right)\left|u_{2}\right|^{2^{*} / 2} .
\end{aligned}
$$


Then, $\left.F_{t_{1}}\right|_{(1,1,1)}=\left.F_{t_{2}}\right|_{(1,1,1)}=\left.F_{s}\right|_{1,1,1}=0$ and

$$
\begin{aligned}
\left.F_{t_{1} t_{1}}\right|_{(1,1,1)}= & || u_{1}^{+} \|_{1}^{2}-\left(p_{1}-1\right) \int v_{1}\left|u_{1}^{+}\right|^{p_{1}}-\left(2^{*}-1\right) \int \mu_{1}\left|u_{1}^{+}\right|^{2^{*}} \\
& -\left(\frac{2^{*}}{2}-1\right) \beta \int\left|u_{1}^{+}\right|^{2^{*} / 2}\left|u_{2}\right|^{2^{*} / 2}
\end{aligned}
$$$$
\left.F_{t_{2} t_{2}}\right|_{(1,1,1)}=\left\|u_{1}^{-}\right\|_{1}^{2}-\left(p_{1}-1\right) \int v_{1}\left|u_{1}^{-}\right|^{p_{1}}-\left(2^{*}-1\right) \int \mu_{1}\left|u_{1}^{-}\right|^{2^{*}}
$$$$
-\left(\frac{2^{*}}{2}-1\right) \beta \int\left|u_{1}^{-}\right|^{2^{*} / 2}\left|u_{2}\right|^{2^{*} / 2}
$$$$
\left.F_{s s}\right|_{(1,1,1)}=\left\|u_{2}\right\|_{2}^{2}-\left.\left(p_{2}-1\right) \int v_{2} u_{2}\right|^{p_{2}}-\left(2^{*}-1\right) \int \mu_{2}\left|u_{2}\right|^{2^{*}}
$$$$
-\left(\frac{2^{*}}{2}-1\right) \beta \int\left|u_{1}\right|^{2^{*} / 2}\left|u_{2}\right|^{2^{*} / 2}
$$$$
\left.F_{t_{1} t_{2}}\right|_{(1,1,1)}=\left.F_{t_{2} t_{1}}\right|_{(1,1,1)}=0 \text {, }
$$$$
\left.F_{t_{1} s}\right|_{(1,1,1)}=\left.F_{s t_{1}}\right|_{(1,1,1)}=-\frac{2^{*}}{2} \beta \int\left|u_{1}^{+}\right|^{2^{*} / 2}\left|u_{2}\right|^{2^{*} / 2},
$$$$
\left.F_{t_{2} s}\right|_{(1,1,1)}=\left.F_{s t_{2}}\right|_{(1,1,1)}=-\frac{2^{*}}{2} \beta \int\left|u_{1}^{-}\right|^{2^{*} / 2}\left|u_{2}\right|^{2^{*} / 2} \text {. }
$$

Set

$$
H_{F}:=\left(\begin{array}{ccc}
F_{t_{1} t_{1}} & F_{t_{1} t_{2}} & F_{t_{1} s} \\
F_{t_{2} t_{1}} & F_{t_{2} t_{2}} & F_{t_{2} s} \\
F_{s t_{1}} & F_{s t_{2}} & F_{s s}
\end{array}\right)_{(1,1,1)} .
$$

Denote

$$
\begin{aligned}
A_{1}^{ \pm} & =\int v_{1}\left|u_{1}^{ \pm}\right|^{p_{1}}, \\
A_{2} & =\int v_{2}\left|u_{2}\right|^{p_{1}}, \\
D_{1}^{ \pm} & =\int \mu_{1}\left|u_{1}^{ \pm}\right|^{2 *}, \\
D_{2} & =\int \mu_{2}\left|u_{2}\right|^{2 *}, \\
D_{1}^{ \pm} & =\int \beta\left|u_{1}^{ \pm}\right|^{2^{*} / 2}\left|u_{2}\right|^{2^{*} / 2}, \\
D & =\int \beta\left|u_{1}\right|^{2^{*} / 2}\left|u_{2}\right|^{2^{*} / 2} .
\end{aligned}
$$

Then,

$$
\begin{aligned}
& a_{11}:=\left.F_{t_{1} t_{1}}\right|_{(1,1,1)}=-\left(p_{1}-2\right) A_{1}^{+}-\left(2^{*}-2\right) D_{1}^{+}-\left(2-\frac{2^{*}}{2}\right) D^{+}<0, \\
& a_{22}:=\left.F_{t_{2} t_{2}}\right|_{(1,1,1)}=-\left(p_{1}-2\right) A_{1}^{-}-\left(2^{*}-2\right) D_{1}^{-}-\left(2-\frac{2^{*}}{2}\right) D^{-}<0, \\
& a_{33}:=\left.F_{s s}\right|_{(1,1,1)}=-\left(p_{2}-2\right) A_{2}-\left(2^{*}-2\right) D_{2}-\left(2-\frac{2^{*}}{2}\right) D<0 .
\end{aligned}
$$

Note that $D=D^{+}+D^{-}, D^{2} \leq\left(D_{1}^{+}+D_{1}^{-}\right) D_{2}$, and $2 D \leq$ $D_{1}^{+}+D_{1}^{-}+D_{2}$. We can show that $H_{F}$ is negative definite. Thus, $(1,1,1)$ is the unique maximum point of $F\left(t_{1}, t_{2}, s\right)$.

Lemma 7. Assume that $-\sqrt{\mu_{1} \mu_{2}}<\beta<0$; then, for any $\mathbf{u} \in \mathbf{H}$ with $u_{1}, u_{2} \equiv 0$, we have that there exist unique $t_{u}, s_{u}>0$ such that $\left(t_{u} u_{1}, s_{u} u_{2}\right) \in \mathscr{M}$. Moreover, $t_{u}$ and $s_{u}$ are continuous with respect to $\mathbf{u}$.

Proof. Proof. For any $\mathbf{u} \in \mathbf{H}$ with $u_{1}, u_{2} \equiv 0$, we denote

$$
\begin{aligned}
E_{1} & =\left\|u_{1}\right\|_{1}^{2}, \\
E_{2} & =\left\|u_{2}\right\|_{2}^{2}, \\
A_{1} & =\int v_{1}\left|u_{1}\right|^{p_{1}}, \\
A_{2} & =\int v_{2}\left|u_{2}\right|^{p_{2},} \\
D_{1} & =\int \mu_{1}\left|u_{1}\right|^{2}, \\
D_{2} & =\int \mu_{2}\left|u_{2}\right|^{2^{*}}, \\
D & =\int \beta\left|u_{1}\right|^{2^{*} / 2}\left|u_{2}\right|^{2^{*} / 2} .
\end{aligned}
$$

Then, $D<0$ and $D_{1} D_{2}>D^{2} .\left(t u_{1}, s u_{2}\right) \in \mathscr{M}$ for some $t, s>0$ is equivalent to

$$
\begin{aligned}
& t^{2} E_{1}=t^{p_{1}} A_{1}+t^{2^{*}} D_{1}+t^{2^{*} / 2} s^{2^{*} / 2} D, \\
& s^{2} E_{2}=s^{p_{2}} A_{2}+s^{2^{*}} D_{2}+t^{2^{*} / 2} s^{2^{*} / 2} D, \quad t, s>0 .
\end{aligned}
$$

By (75), we have

$$
s^{2^{*} / 2}=\frac{t^{2-\left(2^{*} / 2\right)} E_{1}-t^{p_{1}} A_{1}-t^{2^{*}} D_{1}}{D}:=\frac{G(t)}{D}:=\frac{t^{2-\left(2^{*} / 2\right)} \widetilde{G}(t)}{D},
$$

where $s>0$ implies that $\widetilde{G}(t)<0$. Since $\widetilde{G}^{\prime}(t)<0$ and for $t>0$ small enough, $\widetilde{G}(t)>0$, there exists a unique $t_{0}>0$ such that $\widetilde{G}\left(t_{0}\right)=0$; thus, $t>t_{0}$. Combining (76) and (77), it is left to show that

$$
\begin{aligned}
F(t):= & \left(\frac{G(t)}{D}\right)^{\left(4 / 2^{*}\right)-1} t^{-\left(2^{*} / 2\right)} E_{2}-\left(\frac{G(t)}{D}\right)^{\left(2 p_{2} / 2^{*}\right)-1} t^{-\left(2^{*} / 2\right)} A_{2} \\
& -\frac{G(t)}{D} t^{-\left(2^{*} / 2\right)} D_{2}-D,
\end{aligned}
$$

has a solution $t>t_{0}$. Note that $2-\left(2^{*} / 2\right)<p_{1}-\left(2^{*} / 2\right)<$ $\left(2^{*} / 2\right)$ and $4 / 2^{*}-1<\left(2 p_{2} / 2^{*}\right)-1<1$; by direct calculation, we can check that 


$$
\begin{aligned}
& \lim _{t \longrightarrow+\infty} F(t)=\frac{D_{1} D_{2}-D^{2}}{D}<0, \\
& \lim _{t \rightarrow t_{0}} F\left(t_{0}\right)=-D>0 .
\end{aligned}
$$

This implies that (75) and (76) have a positive solution $\left(t_{u}, s_{u}\right)$. The uniqueness of $\left(t_{u}, s_{u}\right)$ follows from Lemma 6 .

For the continuity of $\left(t_{u}, s_{u}\right)$ with respect to $\mathbf{u}$, we take a sequence $\left\{\mathbf{u}_{n}\right\}$ with $\mathbf{u}_{n} \longrightarrow \mathbf{u}$ strongly in $\mathbf{H}$. We may assume that $\left(t_{u}, s_{u}\right)=(1,1)$ by replacing $\mathbf{u}_{n}$ by $\left(t_{u_{n}} \mathbf{u}_{1 n}, s_{u_{n}} \mathbf{u}_{2 n}\right)$ if necessary. That is, $\mathbf{u} \in \mathscr{M}$. Using similar denotations to $(74)$ and rewriting $\left(t_{u_{n}}, s_{u_{n}}\right)=\left(t_{n}, s_{n}\right)$ for convenience, we have

$$
\begin{gathered}
t_{n}^{2} E_{1 n}=t_{n}^{p_{1}} A_{1 n}+t_{n}^{2^{*}} D_{1 n}+t_{n}^{2^{*} / 2} s_{n}^{2^{*} / 2} D_{n}, \\
s_{n}^{2} E_{2}=s_{n}^{p_{2}} A_{2 n}+s_{n}^{2^{*}} D_{2 n}+t_{n}^{2^{*} / 2} s_{n}^{2^{*} / 2} D_{n}, \\
E_{1}=A_{1}+D_{1}+D, E_{2}=A_{2}+D_{2}+D .
\end{gathered}
$$

If $t_{n} \longrightarrow \infty$, then by (81), we have $s_{n} \longrightarrow \infty$. Therefore,

$$
\begin{aligned}
D^{2} & =\lim _{n \longrightarrow+\infty} D_{n}^{2} \\
& =\lim _{n \longrightarrow+\infty} \frac{t_{n}^{2-\left(2^{*} / 2\right)} E_{1 n}-t_{n}^{p_{1}-\left(2^{*} / 2\right)} A_{1 n}-t_{n}^{2^{*} / 2} D_{1 n}}{s_{n}^{2^{*} / 2}} \cdot \frac{s_{n}^{2-\left(2^{*} / 2\right)} E_{2 n}-s_{n}^{p_{2}-\left(2^{*} / 2\right)} A_{2 n}-s_{n}^{2^{*} / 2} D_{2 n}}{t_{n}^{2^{*} / 2}} \\
& =\lim _{n \longrightarrow+\infty}\left(t_{n}^{2-2^{*}} E_{1 n}-t_{n}^{p_{1}-2^{*}} A_{1 n}-D_{1 n}\right)\left(s_{n}^{2-2^{*}} E_{2 n}-s_{n}^{p_{2}-2^{*}} A_{2 n}-D_{2 n}\right) \\
& =\lim _{n \longrightarrow+\infty} D_{1 n} D_{2 n}=D_{1} D_{2}>D^{2},
\end{aligned}
$$

is a contradiction. Thus, $t_{n}$ and $s_{n}$ are uniformly bounded and we may assume that $t_{n} \longrightarrow \hat{t}, s_{n} \longrightarrow \hat{s}$ as $n \longrightarrow+\infty$. By (80) and (81), we have $\left(\hat{t} u_{1}, \hat{s} u_{2}\right) \in \mathscr{M}$. Then, $\hat{t}=\hat{s}=1$, that is, $\left(t_{u_{n}}, s_{u_{n}}\right) \longrightarrow\left(t_{u}, s_{u}\right)$.

Now, we show the following:

Lemma 8. Assume that $-\sqrt{\mu_{1} \mu_{2}}<\beta<0$, then

$$
\mathscr{B}<\min \left\{B+\frac{1}{N} \mu_{1}^{-(N-2 / 2)} S^{N / 2}, \mathscr{B}_{1}+\frac{1}{N} \mu_{2}^{-((N-2) / 2)} S^{N / 2}\right\} .
$$

\section{Proof.}

(i) We first show that $\mathscr{B}<B+(1 / N) \mu_{1}^{-((N-2) / 2)} S^{N / 2}$. In [27], the authors show that (11) has a positive least energy solution; we denote it as $\left(U_{1}, U_{2}\right)$ with the energy

$$
\begin{aligned}
\left\{I\left(U_{1}, U_{2}\right)=\right. & B<\min \left\{\frac{1}{N} \mu_{1}^{-(N-2 / 2)} S^{N / 2}+B_{2}, B_{1}\right. \\
& \left.\left.+\frac{1}{N} \mu_{2}^{-(N-2 / 2)} S^{N / 2}\right\}\right\},
\end{aligned}
$$

and $U_{1}, U_{2} \in C^{2}(\Omega) \cap C(\bar{\Omega})$. For $r>0$ small, take $x_{r} \in \Omega$ such that $\operatorname{di}$ st $\left(x_{r}, \partial \Omega\right)=4 r$ and

$$
\left|U_{1}(x)\right|+\left|U_{2}(x)\right| \leq C r, \quad \forall x \in B\left(x_{r}, 3 r\right) .
$$

Let $\phi \in C_{0}^{\infty}(B(0,2))$ with $0 \leq \phi \leq 1$ and $\phi \equiv 1$ on $B(0,1)$. Set $\phi_{r}(x):=1-\phi\left(\left(x-x_{r}\right) / r\right)$. Then, there exists a positive constant such that $\left|\nabla \phi_{r}(x)\right| \leq(c / r)$. Define $\left(U_{1 r}, U_{2 r}\right):=\left(\phi_{r} U_{1}, \phi_{r} U_{2}\right)$. Then, for $j=1,2$, we have

$$
\begin{aligned}
\int\left|\nabla U_{j r}\right|^{2} & \leq \int\left|\nabla U_{j}\right|^{2}+C r^{N}, \\
\int U_{j r}^{q} & \geq \int U_{j}^{q}-C r^{N+q}, \quad q=2, p_{j}, 2^{*} .
\end{aligned}
$$

By Lemma 7 , there exist $t_{r}, s_{r}>0$ such that $\left(t_{r} U_{1 r}, s_{r} U_{2 r}\right) \in \mathscr{M}$. That is,

$$
\begin{aligned}
t_{r}^{2}\left\|U_{1 r}\right\|_{1}^{2}= & t_{r}^{p} \nu_{1} \int U_{1 r}^{p_{1}}+t_{r}^{2^{*}} \mu_{1} \int U_{1 r}^{2^{*}} \\
& +\beta t_{r}^{2^{*} / 2} s_{r}^{2^{*} / 2} \int U_{1 r}^{2^{*} / 2} U_{2 r}^{2^{*} / 2}
\end{aligned}
$$

$$
\begin{aligned}
s_{r}^{2}\left\|U_{2 r}\right\|_{1}^{2}= & s_{r}^{p} v_{2} \int U_{2 r}^{p_{2}}+s_{r}^{2^{*}} \mu_{1} \int U_{2 r}^{2^{*}} \\
& +\beta t_{r}^{2^{*} / 2} s_{r}^{2^{*} / 2} \int U_{1 r}^{2^{*} / 2} U_{2 r}^{2^{*} / 2} .
\end{aligned}
$$

Then similar to the proof of (83), it can be shown that $t_{r}$ and $s_{r}$ are uniformly bounded. Thus, up to a subsequence, there exist $t_{0}, s_{0}>0$ such that $t_{r} \longrightarrow t_{0}$ and $s_{r} \longrightarrow s_{0}$. Let $r \longrightarrow 0$ in (88) and (89); it implies that $\left(t_{0} U_{1}, s_{0} U_{2}\right) \in \mathscr{M}$. Then, $t_{0}=s_{0}=1$, and we may assume that $(1 / 2) \leq t_{r}$ and $s_{r} \leq 2$ for $r>0$ small. By (87), we have

$$
\begin{aligned}
I\left(t_{r} U_{1 r}, s_{r} U_{2 r}\right) & \leq I\left(t_{r} U_{1}, s_{r} U_{2}\right)+C r^{N} \leq I\left(U_{1}, U_{2}\right)+C r^{N} \\
& =B+C r^{N} .
\end{aligned}
$$

Let $V_{j r}(j=1,2)$ be the solution of problem (8) when $\Omega=B(0, r)$; then, $\left(t_{r} U_{1 r}-V_{1 r}\left(\cdot-x_{r}\right), s_{r} U_{2 r}\right) \in \mathcal{S}$ and from [40], it holds that 


$$
I_{j}\left(V_{j r}\right) \leq \frac{1}{N} \mu_{j}^{-((N-2) / 2)} S^{N / 2}-C_{1} r^{(2 N-4) /(N-4)}, \quad j=1,2 .
$$

Thus,

$$
\begin{aligned}
\mathscr{B} & \leq I\left(t_{r} U_{1 r}-V_{1 r}\left(\cdot-x_{r}\right), s_{r} U_{2 r}\right) \\
& =I\left(t_{r} U_{1 r}, s_{r} U_{2 r}\right)+I_{1}\left(V_{1 r}\left(\cdot-x_{r}\right)\right) \\
& \leq B+C r^{N}+\frac{1}{N} \mu_{1}^{-((N-2) / 2)} S^{N / 2}-C_{1} r^{(2 N-4) /(N-4)} \\
& <B+\frac{1}{N} \mu_{1}^{-((N-2) / 2)} S^{N / 2}, \\
& \text { for } r>0 \text { small. }
\end{aligned}
$$

(ii) Next, we show that $\mathscr{B}<\mathscr{B}_{1}+(1 / N) \mu_{2}^{-(N-2) / 2} S^{N / 2}$. Let $w_{j}$ be the sign changing solution of (8) with $I_{j}\left(w_{j}\right)=\mathscr{B}_{j}$ for $j=1,2$. Then, $w_{j} \in C^{2}(\Omega) \cap C(\bar{\Omega})$ and $\left\{w_{j}^{+}>0\right\}:=\left\{x \in \Omega: w_{j}(x)>0\right\} \neq \varnothing$. For any $R>0$, we may take $y_{R} \in\left\{w_{j}^{+}>0\right\}$ with $\operatorname{dist}\left(y_{R}\right.$, $\left.\partial\left(\left\{w_{j}^{+}>0\right\}\right)\right)=4 R$. Then, $B\left(y_{r}, 3 r\right) \subset\left\{w_{j}^{+}>0\right\}$ and $\left|w_{j}^{+}\right| \leq C R, \forall x \in B\left(y_{R}, 3 R\right)$. Recall $\phi$ and $\phi_{R}$ in the above step (i); we define $U_{1 R}:=\phi_{R} w_{1}^{+}$. Since $I_{1}^{\prime}\left(w_{1}\right) w_{1}^{+}=0$, we have

$$
\left\|w_{1}^{+}\right\|_{1}^{2}=v_{1} \int\left|w_{1}^{+}\right|^{p_{1}}+\mu_{1} \int\left|w_{1}^{+}\right|^{2^{*}} .
$$

Then, there exists $t_{R}>0$ such that

$$
\left\|t_{R} U_{1 R}^{+}\right\|_{1}^{2}=v_{1} \int\left|t_{R} U_{1 R}^{+}\right|^{p_{1}}+\mu_{1} \int\left|t_{R} U_{1 R}^{+}\right|^{2^{*}}
$$

and $I\left(t_{R} U_{1 R}, 0\right)=\max I\left(t U_{1 R}, 0\right)$. It is easy to see that $\left(t_{R} U_{1 R}-w_{1}^{-}, V_{2 R}\left(\cdot{ }^{t>0} y_{R}\right)\right) \in \mathcal{S}$, where $V_{2 R}$ is the positive solution of problem (8) in the ball $B(0, R)$ for $j=2$. Then by (91), we have

$$
\begin{aligned}
& \mathscr{B} \leq I\left(t_{R} U_{1 R}-w_{1}^{-}, V_{2 R}\left(\cdot-y_{R}\right)\right) \\
&= I\left(t_{R} U_{1 R}, 0\right)+I_{1}\left(w_{1}^{-}\right)+I_{2}\left(V_{2 R}\left(\cdot-y_{R}\right)\right) \\
& \leq I_{1}\left(w_{1}^{+}\right)+C R^{N}+I_{1}\left(w_{1}^{-}\right)+\frac{1}{N} \mu_{2}^{-(N-2 / 2)} S^{N / 2} \\
& \quad-C_{1} R^{(2 N-4) / N-4} \\
&< \mathscr{B}_{1}+\frac{1}{N} \mu_{2}^{-(N-2 / 2)} S^{N / 2}, \\
& \text { for } R>0 \text { small. }
\end{aligned}
$$

For $\left(u_{1}, u_{2}\right) \in \mathcal{S}$, we define $u_{1 \rho}:=(1-\rho) u_{1}^{+}-\rho u_{1}^{-}$, $\rho \in[0,1]$. Let $\mathbf{u}_{\rho}:=\left(u_{1 \rho}, u_{2}\right)$ and recall that $\left(t_{u_{\rho}}, s_{u_{\rho}}\right)$ is defined in Lemma 7; then, we can define a continuous map $\eta_{u}:[0,1] \longrightarrow \mathscr{M}$ by

$$
\eta_{u}(\rho):=\left(t_{u_{\rho}} u_{1 \rho}, s_{u_{\rho}} u_{2}\right) .
$$

Then, we have the following conclusion and recall that $\varepsilon_{0}$ is defined in (60).

Lemma 9. For any $\mathbf{u} \in \mathcal{S}$ with $I(\mathbf{u})<\mathscr{B}+\varepsilon_{0}$, there exists a small $\rho_{o} \in(0,(1 / 4))$ such that

$$
\begin{array}{r}
I^{\prime}\left(\eta_{u}\left(\rho_{0}\right)\right)\left(t_{u_{\rho_{0}}} u_{1 \rho_{0}}^{+}, 0\right)<0, \\
I^{\prime}\left(\eta_{u}\left(1-\rho_{0}\right)\right)\left(t_{u_{1-\rho_{0}}} u_{1\left(1-\rho_{0}\right)}^{+}, 0\right)>0, \\
I\left(\eta_{u}\left(\rho_{0}\right)\right), \\
I\left(\eta_{u}\left(1-\rho_{0}\right)\right)<\mathscr{B}-\varepsilon_{0} .
\end{array}
$$

Proof. It is easy to see that there exist constants $C_{1}$ and $C_{2}>0$ such that

$$
\begin{aligned}
& C_{1} \leq t_{u_{\rho}}, \\
& s_{u_{\rho}} \leq C_{2}, \\
& \forall \rho \in[0,1] .
\end{aligned}
$$

Since $I^{\prime}\left(\eta_{u}(\rho)\right)\left(t_{u_{\rho}} u_{1 \rho}, 0\right)=0$ and $u_{1 \rho}^{-}=\rho u_{1}^{-}$, we have

$$
\begin{aligned}
& I^{\prime}\left(\eta_{u}(\rho)\right)\left(t_{u_{\rho}} u_{1 \rho}^{+}, 0\right) \\
& \quad=-I^{\prime}\left(\eta_{u}(\rho)\right)\left(t_{u_{\rho}} \rho u_{1}^{-}, 0\right) \\
& \quad \leq-t_{u_{\rho}}^{2} \rho^{2}\left\|u_{1}^{-}\right\|_{1}^{2}+t_{u_{\rho}}^{p_{1}} \rho^{p_{1}} v_{1} \int\left|u_{1}^{-}\right|^{p_{1}}+t_{u_{\rho}}^{2^{*}} \rho^{2^{*}} \mu_{1} \int\left|u_{1}^{-}\right|^{2^{*}} \\
& \quad \leq C_{1}^{2} \rho^{2} u_{1}^{-2}+C_{2}^{p_{1}} \rho^{p_{1}} v_{1} \int\left|u_{1}^{-}\right|^{p_{1}}+C_{2}^{2^{*}} \rho^{2^{*}} \mu \int\left|u_{1}^{-}\right|^{2^{*}} \\
& \quad<0, \text { for } \rho>0 \text { small. }
\end{aligned}
$$

Similarly, note that $u_{1(1-\rho)}^{+}=\rho u_{1}^{+}$; we have

$$
\begin{aligned}
& I^{\prime}\left(\eta_{u}(1-\rho)\right)\left(t_{u_{1-\rho}} u_{1(1-\rho)}^{+}, 0\right) \\
& \quad \geq t_{u_{1-\rho}}^{2} \rho^{2}\left\|u_{1}^{+}\right\|_{1}^{2}-t_{u_{1-\rho}}^{p_{1}} \rho^{p_{1}} v_{1} \int\left|u_{1}^{+}\right|^{p_{1}}+t_{u_{1-\rho}}^{2^{*}} \rho^{2^{*}} \mu_{1} \int\left|u_{1}^{+}\right|^{2^{*}} \\
& \quad \geq C_{1}^{2} \rho^{2}\left\|u_{1}^{-}\right\|_{1}^{2}-C_{2}^{p_{1}} \rho^{p_{1}} v_{1} \int\left|u_{1}^{-}\right|^{p_{1}}-C_{2}^{2^{*}} \rho^{2^{*}} \mu_{1} \int\left|u_{1}^{-}\right|^{2^{*}} \\
& \quad>0 \text {, for } \rho>0 \text { small. }
\end{aligned}
$$

Since $\mathbf{u} \in \mathcal{S}$, we have $\left(u_{1}^{+}, u_{2}\right),\left(-u_{1}^{-}, u_{2}\right) \in \mathscr{M}$. Then, 


$$
\begin{aligned}
I\left(\eta_{u}(0)\right)= & I\left(u_{1}^{+}, u_{2}\right) \\
= & I\left(u_{1}, u_{2}\right)-\left[\frac{1}{2}\left\|u_{1}^{-}\right\|_{1}^{2}-\frac{v_{1}}{p_{1}} \int\left|u_{1}^{-}\right|^{p_{1}}-\frac{\mu_{1}}{2^{*}} \int\left|u_{1}^{-}\right|^{2^{*}}\right. \\
& \left.+\frac{2|\beta|}{2^{*}} \int\left|u_{1}^{-}\right|^{2^{*} / 2} u_{2}^{2^{*} / 2}\right] \\
= & I\left(u_{1}, u_{2}\right)-\left(\frac{1}{2}-\frac{1}{p_{1}}\right)\left\|u_{1}^{-}\right\|_{1}^{2}-\left[\left(\frac{1}{p_{1}}-\frac{1}{2^{*}}\right) \int \mu_{1}\left|u_{1}^{-}\right|^{2^{*}}\right. \\
& \left.+\left(\frac{2|\beta|}{2^{*}}-\frac{1}{p_{1}}\right) \int\left|u_{1}^{-}\right|^{2^{*} / 2} u_{2}^{2^{*} / 2}\right] \\
\leq & I\left(u_{1}, u_{2}\right)-\left(\frac{1}{2}-\frac{1}{p_{1}}\right)\left\|u_{1}^{-}\right\|_{1}^{2} \\
< & \mathscr{B}-2 \varepsilon_{0} .
\end{aligned}
$$

Similarly, we have

$$
\begin{aligned}
I\left(\eta_{u}(1)\right)= & I\left(-u_{1}^{-}, u_{2}\right) \\
= & I\left(u_{1}, u_{2}\right)-\left[\frac{1}{2} u_{1}^{+2}-\frac{\nu_{1}}{p_{1}} \int\left|u_{1}^{+}\right|^{p_{1}}-\frac{\mu_{1}}{2^{*}} \int\left|u_{1}^{+}\right|^{2^{*}}\right. \\
& \left.+\frac{2|\beta|}{2^{*}} \int\left|u_{1}^{+}\right|^{2^{*} / 2} u_{2}^{2^{*} / 2}\right] \\
\leq & I\left(u_{1}, u_{2}\right)-\left(\frac{1}{2}-\frac{1}{p_{1}}\right) u_{1}^{+2} \\
< & \mathscr{B}-2 \varepsilon_{0} .
\end{aligned}
$$

Since $I^{\prime}$ and $I$ are continuous with respect to $\rho$, (101), (102), (103), and (104) imply (97), (98), and (99), respectively.

Now, we show the existence of a $(\mathrm{PS})_{\mathscr{B}}$ sequence of $I$. Precisely, we have the following important lemma.

Lemma 10. There exist a sequence $\left\{\mathbf{u}_{n}\right\} \subset \mathscr{M}$ and a constant $C>0$ such that

$$
\begin{aligned}
I\left(\mathbf{u}_{n}\right) & \longrightarrow \mathscr{B}, \\
I^{\prime}\left(\mathbf{u}_{n}\right) & \longrightarrow 0, \\
\text { as } n & \longrightarrow+\infty ; \\
\left\|u_{1 n}^{ \pm}\right\|_{1},\left\|u_{2 n}\right\|_{2} & \geq C, \\
\forall n & \geq 1 .
\end{aligned}
$$

Proof. By Lemma 5 and the Ekeland's variational principle, there exists a minimizing sequence $\left\{\widetilde{u}_{n}\right\}=\left\{\left(\widetilde{u}_{1 n}, \widetilde{u}_{2 n}\right)\right\} \subset \mathcal{S}$ such that

$$
\begin{aligned}
& I\left(\widetilde{u}_{n}\right)<\mathscr{B}+\varepsilon_{0}, \\
& I^{\prime}\left(\widetilde{u}_{n}\right) \longrightarrow 0 .
\end{aligned}
$$

Let $\eta_{n}(\rho)=\left(\eta_{n}^{1}(\rho), \eta_{n}^{2}(\rho)\right):=\eta_{\widetilde{u}_{n}}(\rho)$; by Lemma 9 , there exists $\rho_{n} \in(0,(1 / 4))$ such that

$$
\begin{gathered}
I^{\prime}\left(\eta_{n}\left(\rho_{n}\right)\right)\left(\eta_{n}^{1}\left(\rho_{n}\right)^{+}, 0\right)<0, \\
I^{\prime}\left(\eta_{n}\left(1-\rho_{n}\right)\right)\left(\eta_{n}^{1}\left(1-\rho_{n}\right)^{+}, 0\right)>0, \\
I\left(\eta_{n}\left(\rho_{n}\right)\right), \\
I\left(\eta_{n}\left(1-\rho_{n}\right)\right)<\mathscr{B}-\varepsilon_{0} .
\end{gathered}
$$

We claim that there exists a sequence $\left\{\mathbf{u}_{n}\right\} \subset \mathscr{M}$ such that

$$
\begin{aligned}
\operatorname{dist}\left(\mathbf{u}_{n}, \eta_{n}\left(\left[\rho_{n}, 1-\rho_{n}\right]\right)\right) & \longrightarrow 0, \\
\left.I^{\prime}\right|_{\mathscr{M}}\left(u_{n}\right) & \longrightarrow 0, \\
I\left(u_{n}\right) & \longrightarrow \mathscr{B} .
\end{aligned}
$$

If not, then we assume that there exists small $\varepsilon_{1}>0$ such that

$$
\forall \mathbf{u} \in I_{\mathscr{M}}^{-1}\left(\left[\mathscr{B}-\varepsilon_{1}, \mathscr{B}+\varepsilon_{1}\right]\right) \cap S_{\varepsilon_{1}}:\left\|\left.I\right|_{\mathscr{M}} ^{\prime}(\mathbf{u})\right\| \geq \varepsilon_{1},
$$

where

$$
\begin{aligned}
\left.I\right|_{\mathscr{M}} ^{-1}\left(\left[\mathscr{B}-\varepsilon_{1}, \mathscr{B}+\varepsilon_{1}\right]\right) & :=\left\{\mathbf{u} \in \mathscr{M}: I(\mathbf{u}) \in\left[\mathscr{B}-\varepsilon_{1}, \mathscr{B}+\varepsilon_{1}\right]\right\}, \\
S & :=\eta_{n}\left(\left[\rho_{n}, 1-\rho_{n}\right]\right),
\end{aligned}
$$

and $S_{\varepsilon_{1}}$ is the $\varepsilon_{1}$ neighborhood of $S$. Then, by the well-known deformation lemma, there exist a continuous map $\xi:[0,1] \times$ $\mathscr{M} \longrightarrow \mathscr{M}$ and an $\varepsilon_{2} \in\left(0,\left(\min \left\{\varepsilon_{0}, \varepsilon_{1}\right\}\right) / 4\right)$ such that

(1) $\xi(0, \mathbf{u})=\mathbf{u}, \forall \mathbf{u} \in \mathscr{M}$

(2) $\xi(t, \mathbf{u})=\mathbf{u}, \forall \mathbf{u} \notin I^{-1}\left(\left[\mathscr{B}-2 \varepsilon_{2}, \mathscr{B}+2 \varepsilon_{2}\right]\right), t \in[0,1]$

(3) $\left.\xi\left(1,\left.I\right|_{\mathscr{M}} ^{\mathscr{B}+\varepsilon_{2}} \cap S\right) \subset I\right|_{\mathscr{M}} ^{\mathscr{B}-\varepsilon_{2}}$

By Lemma 6, for $n$ large enough, we have that

$$
\sup _{\rho \in\left[\rho_{n}, 1-\rho_{n}\right]} I\left(\eta_{n}(\rho)\right) \leq I\left(\widetilde{u}_{n}\right)<\mathscr{B}+\varepsilon_{2} .
$$

Then, by the properties (2) and (3) of $\xi$ and (109), we have

$$
\sup _{\rho \in\left[\rho_{n}, 1-\rho_{n}\right]} I\left(\xi\left(1, \eta_{n}(\rho)\right)\right) \leq \mathscr{B}-\varepsilon_{2},
$$

$$
\begin{aligned}
\xi\left(1, \eta_{n}\left(\rho_{n}\right)\right) & =\eta_{n}\left(\rho_{n}\right), \\
\xi\left(1, \eta_{n}\left(1-\rho_{n}\right)\right) & =\eta_{n}\left(1-\rho_{n}\right) .
\end{aligned}
$$

Similarly as in (20), we define 


$$
E\left(u_{1}, u_{2}\right):= \begin{cases}0, & u_{1}=0, \\ \frac{v_{1} \int\left|u_{1}\right|^{p_{1}}+\mu_{1} \int\left|u_{1}\right|^{2^{*}}+\beta \int\left|u_{1}\right|^{2^{*} / 2}\left|u_{2}\right|^{2^{*} / 2}}{\left\|u_{1}\right\|_{1}^{2}}, & u_{1} \neq 0 .\end{cases}
$$

We rewrite $\eta(\rho):=\left(\eta^{1}(\rho), \eta^{2}(\rho)\right):=\xi\left(1, \eta_{n}(\rho)\right)$ for convenience. $\eta(\rho) \in \mathscr{M}$ implies that $E\left(\left(\eta^{1}(\rho)\right), \eta^{2}(\rho)\right)=1$. By (107) and (108), we have that

$$
\begin{aligned}
E\left(\left(\eta^{1}\left(\rho_{n}\right)\right)^{+}, \eta^{2}\left(\rho_{n}\right)\right) & >1, \\
E\left(\left(\eta^{1}\left(1-\rho_{n}\right)\right)^{+}, \eta^{2}\left(1-\rho_{n}\right)\right) & <1 .
\end{aligned}
$$

Then, by the continuity of $E$, we can define

$$
\rho_{0}:=\max \left\{\rho \in\left[\rho_{n}, 1-\rho_{n}\right]: E\left(\eta^{1}(\rho)^{+}, \eta^{2}(\rho)\right)=1\right\} .
$$

By definition of $\rho_{0}$, there exists $\delta_{0} \in\left(0,1-\rho_{n}-\rho_{0}\right)$ such that $E\left(\eta^{1}(\rho)^{+}, \eta^{2}(\rho)\right)<1$ for any $\rho \in\left(\rho_{0}, \rho_{0}+\delta_{0}\right)$. Then, $E\left(\eta^{1}(\rho)^{-}, \eta^{2}(\rho)\right)>1$ for any $\rho \in\left(\rho_{0}, \rho_{0}+\delta_{0}\right)$. Thus, $E\left(\eta^{1}\left(\rho_{0}\right)^{-}, \eta^{2}\left(\rho_{0}\right)\right) \geq 1$. Hence, $E\left(\eta^{1}\left(\rho_{0}\right)^{ \pm}, \eta^{2}\left(\rho_{0}\right)\right)=1$. That is, $\eta\left(\rho_{0}\right) \in \mathcal{S}$. But by (115), we have $I\left(\eta\left(\rho_{0}\right)\right)<\mathscr{B}$, a contradiction with the definition of $\mathscr{B}$. Therefore, the claim (111) becomes true.

Now, we can choose $\hat{\rho}_{n} \in\left[\rho_{n}, 1-\rho_{n}\right]$ such that $\| \mathbf{u}_{n}-$ $\eta_{n}\left(\hat{\rho}_{n}\right) \| \longrightarrow 0, n \longrightarrow \infty$. Since $I\left(\mathbf{u}_{n}\right) \longrightarrow \mathscr{B}$, we have $I\left(\eta_{n}\left(\hat{\rho}_{n}\right)\right) \longrightarrow \mathscr{B}$. Recall that $\widetilde{u}_{n} \in \mathcal{S}$ and $I\left(\widetilde{u}_{n}\right) \longrightarrow \mathscr{B}$; then by Lemma 6 , we have $\left\|\eta_{n}\left(\hat{\rho}_{n}\right)-\widetilde{u}_{n}\right\| \longrightarrow 0$. By (59), we have that there exists a constant $C>0$ independent of $n$ such that $\left\|u_{1 n}^{ \pm}\right\|_{1},\left\|u_{2 n}\right\|_{2} \geq C>0$.

Set

$$
\begin{aligned}
& J_{1}\left(u_{1}, u_{2}\right):=I^{\prime}\left(u_{1}, u_{2}\right)\left(u_{1}, 0\right), \\
& J_{2}\left(u_{1}, u_{2}\right):=I^{\prime}\left(u_{1}, u_{2}\right)\left(0, u_{2}\right) .
\end{aligned}
$$

By (111), there exist two Lagrange multipliers $L_{1 n}, L_{2 n} \in \mathbb{R}$ such that

$$
\begin{aligned}
J\left(u_{1 n}, u_{2 n}\right):= & I^{\prime}\left(u_{1 n}, u_{2 n}\right)+L_{1 n} J_{1}^{\prime}\left(u_{1 n}, u_{2 n}\right) \\
& +L_{2 n} J_{2}^{\prime}\left(u_{1 n}, u_{2 n}\right) \longrightarrow 0 .
\end{aligned}
$$

Then, $\quad\left(J\left(u_{1 n}, u_{2 n}\right),\left(u_{1 n}, 0\right)\right)=o(1)$ $\left(J\left(u_{1 n}, u_{2 n}\right),\left(0, u_{2 n}\right)\right)=o(1)$; i.e.,

$$
\begin{aligned}
& L_{1 n}\left[\left(p_{1}-2\right) \int v_{1}\left|u_{1 n}\right|^{p_{1}}+\left(2^{*}-2\right) \int \mu_{1}\left|u_{1 n}\right|^{2^{*}}+\left(2-\frac{2^{*}}{2}\right) \int|\beta|\left|u_{1 n}\right|^{2^{*} / 2}\left|u_{2 n}\right|^{2^{*} / 2}\right] \\
& \quad=L_{2 n} \frac{2^{*}}{2} \int|\beta|\left|u_{1 n}\right|^{2^{*} / 2}\left|u_{2 n}\right|^{2^{*} / 2}+o(1), \\
& L_{2 n}\left[\left(p_{2}-2\right) \int v_{2}\left|u_{2 n}\right|^{p_{2}}+\left(2^{*}-2\right) \int \mu_{2}\left|u_{2 n}\right|^{2^{*}}+\left(2-\frac{2^{*}}{2}\right) \int|\beta|\left|u_{1 n}\right|^{2^{*} / 2}\left|u_{2 n}\right|^{2^{*} / 2}\right] \\
& \quad=L_{1 n} \frac{2^{*}}{2} \int|\beta|\left|u_{1 n}\right|^{2^{*} / 2}\left|u_{2 n}\right|^{2^{*} / 2}+o(1) .
\end{aligned}
$$

Similarly as in (74), we denote

$$
\begin{aligned}
A_{1 n} & =\int v_{1}\left|u_{1 n}\right|^{p_{1}}, \\
A_{2 n} & =\int v_{2}\left|u_{2 n}\right|^{p_{2}}, \\
D_{1 n} & =\int \mu_{1}\left|u_{1 n}\right|^{2^{*}}, \\
D_{2 n} & =\int \mu_{2}\left|u_{2 n}\right|^{2^{*}}, \\
D_{n} & =\int|\beta|\left|u_{1 n}\right|^{2^{*} / 2}\left|u_{2 n}\right|^{2^{*} / 2} .
\end{aligned}
$$

Note that $\left(u_{1 n}, u_{2 n}\right) \subset \mathscr{M}$, we have

$$
\begin{aligned}
& \left\|u_{1 n}\right\|_{1}^{2}=A_{1 n}+D_{1 n}-D_{n}, \\
& \left\|u_{2 n}\right\|_{2}^{2}=A_{2 n}+D_{2 n}-D .
\end{aligned}
$$

Consequently, by (59), we have

$$
\begin{gathered}
A_{1 n}+D_{1 n}>D_{n}, \\
A_{2 n}+D_{2 n}>D .
\end{gathered}
$$

Set

$$
\begin{aligned}
S_{1 n}: & =\left(p_{1}-2\right)\left(p_{2}-2\right) A_{1 n} A_{2 n}+\left(p_{1}-2\right)\left(2^{*}-2\right) A_{1 n} D_{2 n} \\
& +\left(p_{1}-2\right)\left(2-\frac{2^{*}}{2}\right) A_{1 n} D_{n}+\left(p_{2}-2\right)\left(2^{*}-2\right) A_{2 n} D_{1 n} \\
& +\left(p_{2}-2\right)\left(2-\frac{2^{*}}{2}\right) A_{2 n} D_{n}, \\
S_{2 n}:= & \left(2^{*}-2\right)^{2} D_{1 n} D_{2 n}+\left(2^{*}-2\right)\left(2-\frac{2^{*}}{2}\right) D_{1 n} D_{n} \\
& +\left(2^{*}-2\right)\left(2-\frac{2^{*}}{2}\right) D_{2 n} D_{n}-2\left(2^{*}-2\right) D_{n}^{2} .
\end{aligned}
$$

Then, by (122) and (123), we have

$$
o(1)=L_{1 n} L_{2 n}\left(S_{1 n}+S_{2 n}\right):=L_{1 n} L_{2 n} S_{n} .
$$

Note that 


$$
\begin{aligned}
& D_{n} \leq \frac{1}{2}\left(D_{1 n}+D_{2 n}\right), \\
& D_{n}^{2} \leq D_{1 n} D_{2 n} .
\end{aligned}
$$

We have

$$
S_{2 n} \geq\left(2^{*}-2\right)^{2}\left(D_{1 n} D_{2 n}-D_{n}^{2}\right) \geq 0 .
$$

Thus, $S_{n}>C>0$ for some constant $C>0$. By (128), we have $L_{1 n} L_{2 n}=o(1)$. Without loss of generality, we may assume that $L_{1 n}=o(1)$; then by (123) and (59), we have $L_{2 n}=o(1)$. Then, (121) implies that $I^{\prime}\left(u_{1 n}, u_{2 n}\right) \longrightarrow 0$. Thus, we complete the proof.

We also need an important lemma which is proved in [25].

Lemma 11 (see [25]). Assume that $\left(u_{1 n}, u_{2 n}\right) \rightarrow\left(u_{1}, u_{2}\right)$ weakly in $\mathbf{H}$ as $n \longrightarrow \infty$; then, passing to a subsequence, it holds that

$$
\begin{gathered}
\lim _{n \rightarrow \infty} \int\left(\left|u_{1 n}\right|^{2^{*} / 2}\left|u_{2 n}\right|^{2^{*} / 2}-\left|u_{1}\right|^{2^{*} / 2}\left|u_{2}\right|^{2^{*} / 2}\right. \\
\left.-\left|u_{1 n}-u_{1}\right|^{2^{*} / 2}\left|u_{2 n}-u_{2}\right|^{2^{*} / 2}\right)=0
\end{gathered}
$$

Proof of Theorem 3. Proof of Theorem 3. Let $\left\{\mathbf{u}_{n}\right\} \subset \mathscr{M}$ be the sequence obtained in Lemma 10. Then, up to a subsequence, there exists $\mathbf{u} \in \mathbf{H}$ such that $\mathbf{u}_{n} \rightarrow \mathbf{u}$ weakly in H. Then, $I^{\prime}(\mathbf{u})=0$ and

$$
\left\|u_{1 n}\right\|_{1}^{2}=\int\left(v_{1}\left|u_{1 n}\right|^{p_{1}}+\mu_{1}\left|u_{1 n}\right|^{2^{*}}+\beta\left|u_{1 n}\right|^{2^{*} / 2}\left|u_{2 n}\right|^{2^{*} / 2}\right)
$$

$$
\left\|u_{2 n}\right\|_{2}^{2}=\int\left(v_{2}\left|u_{2 n}\right|^{p_{2}}+\mu_{2}\left|u_{1 n}\right|^{2^{*}}+\beta\left|u_{1 n}\right|^{2^{*} / 2}\left|u_{2 n}\right|^{2^{*} / 2}\right),
$$

$$
\left\|u_{1 n}^{ \pm}\right\|_{1}^{2}=\int\left(v_{1}\left|u_{1 n}^{ \pm}\right|^{p_{1}}+\mu_{1}\left|u_{1 n}^{ \pm}\right|^{2^{*}}+\beta\left|u_{1 n}^{ \pm}\right|^{2^{*} / 2}\left|u_{2 n}\right|^{2^{*} / 2}\right)+o(1) .
$$

Set $\mathbf{w}_{n}=\left(w_{1 n}, w_{2 n}\right):=\mathbf{u}_{n}-\mathbf{u}$. By Lemma 11, (133), and (134), we have

$$
\begin{gathered}
\int\left|\nabla\left(w_{1 n}^{ \pm}\right)\right|^{2}=\int\left(\mu_{1}\left|w_{1 n}^{ \pm}\right|^{2^{*}}+\beta\left|w_{1 n}^{ \pm}\right|^{2^{*} / 2}\left|w_{2 n}\right|^{2^{*} / 2}\right)+o(1) \\
\int\left|\nabla w_{2 n}\right|^{2}=\int\left(\mu_{2}\left|w_{2 n}\right|^{2^{*}}+\beta\left|w_{1 n}\right|^{2^{*} / 2}\left|w_{2 n}\right|^{2^{*} / 2}\right)+o(1) .
\end{gathered}
$$

Then,

$$
\begin{aligned}
\mathscr{B} & =\lim _{n \longrightarrow \infty} I\left(\mathbf{u}_{n}\right)=\lim _{n \longrightarrow \infty}\left[\frac{1}{N}\left\|\mathbf{u}_{n}\right\|^{2}+\int \sum_{j}\left(\frac{1}{2^{*}}-\frac{1}{p_{j}}\right) v_{j}\left|u_{j n}\right|^{p_{j}}\right] \\
& =\left[\frac{1}{N}\|\mathbf{u}\|^{2}+\int \sum_{j}\left(\frac{1}{2^{*}}-\frac{1}{p_{j}}\right) v_{j}\left|u_{j}\right|^{p_{j}}\right]+\lim _{n \longrightarrow \infty} \frac{1}{N} \int\left(\left|\nabla\left(w_{1 n}^{+}\right)\right|^{2}+\left|\nabla\left(w_{1 n}^{-}\right)\right|^{2}+\left|\nabla w_{2 n}\right|^{2}\right) .
\end{aligned}
$$

Case 1. 1. Assume that $u_{1}, u_{2} \equiv 0$. Then, $u_{j n} \longrightarrow 0$ in $L^{2}(\Omega)$ and $L^{p_{j}}(\Omega)$ for $j=1,2$. From (135) and (136), we have

$$
\begin{gathered}
\int\left|\nabla\left(w_{1 n}^{ \pm}\right)\right|^{2} \leq \mu_{1} S^{-\left(2^{*} / 2\right)}\left(\int\left|\nabla\left(w_{1 n}^{ \pm}\right)\right|^{2}\right)^{2^{*} / 2}, \\
\int\left|\nabla w_{2 n}\right|^{2} \leq \mu_{2} S^{-\left(2^{*} / 2\right)}\left(\int\left|\nabla w_{2 n}\right|^{2}\right)^{2^{*} / 2} .
\end{gathered}
$$

Then,

$$
\begin{gathered}
\int\left|\nabla\left(w_{1 n}^{ \pm}\right)\right|^{2} \geq \mu_{1}^{-((N-2) / 2)} S^{N / 2}, \\
\int\left|\nabla w_{2 n}\right|^{2} \geq \mu_{2}^{-((N-2) / 2)} S^{N / 2} .
\end{gathered}
$$

Thus, by (137), we have

$$
\mathscr{B} \geq \frac{2}{N} \mu_{1}^{-((N-2) / 2)} S^{N / 2}+\frac{1}{N} \mu_{2}^{-(N-2 / 2)} S^{N / 2},
$$

a contradiction with Lemma 8, (85), and (9). Therefore, Case 1 is impossible.
Case 2. Assume that $u_{1} \equiv 0$ and $u_{2} \equiv 0$. Then, (139) still holds and $I^{\prime}\left(0, u_{2}\right)=0$ which implies that $u_{2}$ is a nontrivial solution of (10) with $j=2$. Thus, $I\left(0, u_{2}\right) \geq B_{2}$. By (137), we have

$$
\mathscr{B} \geq B_{2}+\frac{2}{N} \mu_{1}^{-(N-2 / 2)} S^{N / 2},
$$

a contradiction with Lemma 8 and (85). Therefore, Case 2 is impossible.

Case 3. Assume that $u_{1} \equiv 0$ and $u_{2} \equiv 0$. Then, (140) still holds and $I^{\prime}\left(u_{1}, 0\right)=0$ which implies that $u_{1}$ is a nontrivial solution of (10) with $j=1$. Thus, $I\left(u_{1}, 0\right) \geq B_{1}$.

(i) If $u_{1 n} \longrightarrow u_{1}$ strongly in $H_{0}^{1}(\Omega)$, then $u_{1}$ is a sign changing solution of $(10)$ with $j=1$. So $I\left(u_{1}, 0\right) \geq \mathscr{B}_{1}$. By (137), we have

$$
\mathscr{B} \geq \mathscr{B}_{1}+\frac{1}{N} \mu_{2}^{-((N-2) / 2)} S^{N / 2},
$$

a contradiction with Lemma 8 . 
(ii) If $u_{1 n} \nrightarrow u_{1}$ strongly in $H_{0}^{1}(\Omega)$, then by (132) and Brezis-Lieb lemma, we can also show that

$$
\int\left|\nabla w_{1 n}\right|^{2} \geq \mu_{1}^{-((N-2) / 2)} S^{N / 2}
$$

By (137), we have

$$
\mathscr{B} \geq B_{1}+\frac{1}{N} \mu_{1}^{-((N-2) / 2)} S^{N / 2}+\frac{1}{N} \mu_{2}^{-((N-2) / 2)} S^{N / 2},
$$

a contradiction with Lemma 8 and (85).

Therefore, Case 3 is impossible.

Now, we have shown that $u_{1}, u_{2} \equiv 0$ and then $\left(u_{1}, u_{2}\right)$ is a nontrivial solution of (11). If $u_{1 n} \nrightarrow u_{1}$ strongly in $H_{0}^{1}(\Omega)$, then (144) holds and

$$
\mathscr{B} \geq B+\frac{1}{N} \mu_{1}^{-((N-2) / 2)} S^{N / 2}
$$

a contradiction with Lemma 8 . Thus, $u_{1 n} \longrightarrow u_{1}$ strongly in $H_{0}^{1}(\Omega)$ and then $u_{1}$ is sign changing, $I(\mathbf{u}) \geq \mathscr{B}$. On the other hand, by (137), we have

$$
I(\mathbf{u}) \leq \lim _{n \longrightarrow \infty} I\left(\mathbf{u}_{n}\right)=\mathscr{B} .
$$

Then, we have $I(\mathbf{u})=\mathscr{B}$ and $\mathbf{u}_{n} \longrightarrow \mathbf{u}$ strongly in $\mathbf{H}$. Then $\left(u_{1},\left|u_{2}\right|\right) \in \mathcal{S}$ and $I\left(u_{1},\left|u_{2}\right|\right)=\mathscr{B}$. Similarly to the proof in Lemma 10 , we have $I^{\prime}\left(u_{1},\left|u_{2}\right|\right)=0$. By the maximum principle, we have $\left|u_{2}\right|>0$ in $\Omega$. Consequently, $\left(u_{1},\left|u_{2}\right|\right)$ is a least energy sign changing solution of system (11) with $u_{1}$ sign changing and $u_{2}$ positive. Thus, we complete the proof.

\section{Conclusion}

In this paper, we study a coupled nonlinear Schrödinger system with critical exponents which arise in many physical problems. By the modified Nehari manifold method and some mathematical skills for estimations of the energy, we show the existence of the least energy sign changing solution for the general system. Besides, we construct multiple solutions of the system for the symmetrical case.

\section{Data Availability}

No data were used to support this study and all materials are available in this study.

\section{Conflicts of Interest}

The authors declare that there are no conflicts of interest.

\section{Authors' Contributions}

All authors studied this problem and read and approved the final manuscript.

\section{Acknowledgments}

This work was supported by the NSFC (11601109), Hainan Association of Science and Technology plans for Youth Innovation (201503), and Natural Science Fund of Hainan Province (20161001).

\section{References}

[1] N. Akhmediev and A. Ankiewicz, "Partially coherent solitons on a finite background," Physical Review Letters, vol. 82, no. 13, pp. 2661-2664, 1999.

[2] B. Sirakov, "Least energy solitary waves for a system of a nonlinear Schrödinger equations in $\mathbb{R}^{n}$," Communications in Mathematical Physics, vol. 271, no. 1, pp. 199-221, 2007.

[3] B. D. Esry, C. H. Greene, J. P. Burke Jr., and J. L. Bohn, "Hartree-fock theory for double condesates," Physical Review Letters, vol. 78, no. 19, pp. 3594-3597, 1997.

[4] A. Ambrosetti and E. Colorado, "Standing waves of some coupled nonlinear Schrödinger equations," Journal of the London Mathematical Society, vol. 75, no. 1, pp. 67-82, 2007.

[5] T. Bartsch, N. Dancer, and Z.-Q. Wang, "A Liouville theorem, a priori bounds and bifurcating branches of positive solutions for a nonlinear elliptic system," Calculus of Variations and Partial Differential Equations, vol. 37, no. 3-4, pp. 345-361, 2010.

[6] T. Bartsch and Z.-Q. Wang, "Note on ground states of nonlinear Schrödinger systems," Journal of Partial Differential Equations, vol. 19, pp. 200-207, 2006.

[7] T. Bartsch, Z.-Q. Wang, and J. Wei, "Bound states for a coupled Schrödinger system," Journal of Fixed Point Theory and Applications, vol. 2, no. 2, pp. 353-367, 2007.

[8] Z. Chen, C.-S. Lin, and W. Zou, "Infinitely many signchanging and semi-nodal solutions for a nonlinear Schrödinger system," Annali della Scuola Normale Superiore di Pisa, vol. 15, pp. 859-897, 2016.

[9] Z. Chen, C.-S. Lin, and W. Zou, "Multiple sign-changing and semi-nodal solutions for coupled Schrödinger equations," Journal of Differential Equations, vol. 255, no. 11, pp. 42894311, 2013.

[10] Z. Chen and W. Zou, "An optimal constant for the existence of least energy solutions of a coupled Schrödinger system," Calculus of Variations and Partial Differential, vol. 48, no. 3-4, pp. 695-711, 2013.

[11] M. Conti, S. Terracini, and G. Verzini, "Asymptotic estimates for the spatial segregation of competitive systems," Advances in Mathematics, vol. 195, no. 2, pp. 524-560, 2005.

[12] M. Conti, S. Terracini, and G. Verzini, "Nehari's problem and competing species systems," Annales de l'Institut Henri Poincare (C) Non Linear Analysis, vol. 19, no. 6, pp. 871-888, 2002.

[13] E. N. Dancer, J.-C. Wei, and T. Weth, “A priori bounds versus multiple existence of positive solutions for a nonlinear Schrödinger system," Annales de l'Institut Henri Poincare (C) Non Linear Analysis, vol. 27, no. 3, pp. 953-969, 2010.

[14] T.-C. Lingua and J. Wei, "Ground state of N-coupled nonlinear Schrödinger equations in $\mathbb{R}^{N}, N \leq 3$," Communications in Mathematical Physics, vol. 255, no. 3, pp. 629-653, 2005.

[15] T.-C. Lin and J.-C. Wei, "Spikes in two coupled nonlinear Schrödinger equations," Annales de l'Institut Henri Poincare (C) Non Linear Analysis, vol. 22, no. 4, pp. 403-439, 2005. 
[16] L. A. Maia, E. Montefusco, and B. Pellacci, "Infinitely many nodal solutions for a weakly coupled nonlinear Schrödinger system," Communications in Contemporary Mathematics, vol. 10 , no. 05 , pp. 651-669, 2008.

[17] B. Noris and M. Ramos, "Existence and bounds of positive solutions for a nonlinear Schrödinger system," Proceedings of the American Mathematical Society, vol. 138, no. 5, pp. 1681-1692, 2010.

[18] B. Noris, S. Terracini, H. Tavares, and G. Verzini, "Uniform hölder bounds for nonlinear Schrödinger systems with strong competition," Communications on Pure and Applied Mathematics, vol. 63, no. 3, pp. 267-302, 2010.

[19] Y. Sato and Z.-Q. Wang, "On the least energy sign-changing solutions for a nonlinear elliptic sytem," Discrete and Continuous Dynamical Systems, vol. 35, no. 5, pp. 2151-2164, 2014.

[20] H. Tavares and S. Terracini, "Sign-changing solutions of competition-diffusion elliptic systems and optimal partition problems," Annales de l'Institut Henri Poincare (C) Non Linear Analysis, vol. 29, no. 2, pp. 279-300, 2012.

[21] S. Terracini and G. Verzini, "Multipulse phases in k-mixtures of Bose-Einstein condensates," Archive for Rational Mechanics and Analysis, vol. 194, no. 3, pp. 717-741, 2009.

[22] J. Wei and T. Weth, "Radial solutions and phase separation in a system of two coupled Schrödinger equations," Archive for Rational Mechanics and Analysis, vol. 190, no. 1, pp. 83-106, 2008.

[23] X. Yue, "Positive ground state solutions and multiple nontrivial solutions for coupled critical elliptic systems," Journal of Mathematical Analysis and Applications, vol. 427, no. 1, pp. 88-106, 2015.

[24] Z. Chen and W. Zou, "Positive least energy solutions and phase separation for coupled nonlinear Schrödinger equations with critical exponent," Archive for Rational Mechanics and Analysis, vol. 205, no. 2, pp. 515-551, 2012.

[25] Z. Chen and W. Zou, "Positive least energy solutions and phase separation for coupled Schrödinger equations with critical exponent: higher dimensional case," Calculus of Variations and Partial Differential Equations, vol. 52, no. 1-2, pp. 423-467, 2015.

[26] S. Kim, "On vector solutions for coupled nonlinear Schrödinger equations with critical exponents," Communications on Pure \& Applied Analysis, vol. 12, no. 3, pp. 12591277, 2013.

[27] Z. Chen, C.-S. Lin, and W. Zou, "Sign-changing solutions and phase seperation for an elliptic system with critical exponent," Communications in Partial Differential Equations, vol. 39, no. 10, pp. 1827-1859, 2014.

[28] H. Brezis and L. Nirenberg, "Positive solutions of nonlinear elliptic equations involving critical Sobolev exponent," Communications on Pure and Applied Mathematics, vol. 36, no. 4, pp. 437-477, 1983.

[29] G. Cerami, S. Solimini, and M. Struwe, "Some existence results for superlinear elliptic boundary value problems involving critical exponents," Journal of Functional Analysis, vol. 69 , no. 3, pp. 289-306, 1986.

[30] A. Ambrosetti and M. Struwe, "A note on the problem $-\Delta u=\lambda u+u|u|^{2^{*}-2}, "$ Manuscripta Mathematica, vol. 54, no. 4, pp. 373-379, 1986.

[31] A. Capozzi, D. Fortunato, and G. Palmieri, "An existence result for nonlinear elliptic problems involving critical Sobolev exponent," Annales de l'Institut Henri Poincare (C) Non Linear Analysis, vol. 2, no. 6, pp. 463-470, 1985.

[32] C. Miranda, "Un'osservazione sul teorem di Brouwer," Bolletino della Unione Matematica Italiana Serie II, vol. 3, pp. 5-7, 1940.
[33] T. Aubin, "Problems isoperimetriques et espaces de Sobolev," Journal of Differential Geometry, vol. 11, no. 4, pp. 573-598, 1976.

[34] G. Talenti, "Best constants in Sobolev inequality," Annali di Matematica Pura ed Applicata, vol. 110, no. 1, pp. 352-372, 1976.

[35] M. Willem, Minimax Theorems, Birkhäuser, Basel, Switzerland, 1996.

[36] A. Ambrosetti and P. H. Rabinowitz, "Dual variational methods in critical point theory and applications," Journal of Functional Analysis, vol. 14, no. 4, pp. 349-381, 1973.

[37] R. Nadezhda, T. Tsvetomir, and K. Mikhail, "Deformation lemma, ljusternik-Schnirellmann theory and mountain pass theorem on $C^{1}$-finsler manifolds," Serdica Mathematical Journal, vol. 21, pp. 239-266, 1995.

[38] S. Solimini, "On the solvability of some elliptic partial differential equations with the linear part at resonance," Journal of Mathematical Analysis and Applications, vol. 117, no. 1, pp. 138-152, 1986.

[39] X. Yue and W. Zou, "Remarks on a Brezis-Nirenberg's result," Journal of Mathematical Analysis and Applications, vol. 425, no. 2, pp. 900-910, 2015.

[40] Z. Chen and W. Zou, "On the Brezis-Nirenberg problem in a ball," Differential Integral Equations, vol. 25, no. 5-6, pp. 527-542, 2012. 